\title{
35. STABLE CARBON AND NITROGEN ISOTOPE COMPOSITIONS OF ORGANIC MATTER FROM SITES 724 AND 725, OMAN MARGIN1
}

\author{
Alfred N. N. Muzuka, ${ }^{2}$ Stephen A. Macko, ${ }^{2,4}$ and T. F. Pedersen ${ }^{3}$
}

\begin{abstract}
Stable isotopic compositions of carbon and nitrogen and organic carbon content of sediments ranging from the Pliocene to the Pleistocene-Holocene in age from the Oman Margin (ODP Sites 724 and 725) are reported. In general, the organic carbon content is greater than $2 \%$ at Site $\mathbf{7 2 4}$. Prior to the Pleistocene-Holocene at this site, sediments with higher content of organic matter were deposited owing to favorable preservation conditions and/or higher productivity. In the Pleistocene, lower amounts of organic matter have been preserved; this material generally has more enriched nitrogen isotopic compositions. This may indicate intensification of the Oxygen Minimum Zone and denitrification with the onset of the Pleistocene. A correlation of carbon isotope content of these sediments with oxygen isotope stages at Site 724 indicates an enrichment in ${ }^{13} \mathrm{C}$ during glacial events. Based on the stable isotope evidence of both carbon and nitrogen, there does not appear to be major input of terrigenous-derived allochthonous material in this marine environment. The timing and extent of monsoon winds on the productivity of this region are not evident, but require further studies for collaborative interpretation of small-scale features in the isotopic and carbon content of this environment.
\end{abstract}

\section{INTRODUCTION}

One of the primary goals of drilling in the western Arabian Sea during Ocean Drilling Project Leg 117 was to determine the history of monsoon variability by studying the fluctuations in oceanic circulation and productivity as preserved in the sedimentary record. The effects of land mass altitude, atmospheric circulation, and glacial events on the initiation and magnitude of the monsoons need to be incorporated into paleoclimatic models. Holes were drilled at 12 sites on the Indus Fan, the Owen Ridge, and the Oman Margin. This paper presents stable carbon and nitrogen isotope analyses on the sediment organic matter from two of the Oman Margin sites (Fig. 1).

The sites which were studied, 724 and 725 , are located on the continental margin of Oman in water depths of $592.8 \mathrm{~m}$ and $311.5 \mathrm{~m}$, respectively. In addition to the primary objectives of this leg, these sites were drilled to obtain high resolution information to establish the relationships among the intensity and timing of monsoons, the productivity of the region, and the fluctuation and effects of the oxygen minimum zone (OMZ) on the preservation and diagenesis of organic matter in sediments below an upwelling area.

The amount and type of organic matter produced in the upwelling zone is a product of oceanic circulation, water column concentrations of nutrients, and the hydrographic regime (Zahn and Pedersen, this volume). The amount of organic matter produced over time should be related to the development of regional upwelling, which depends directly on the intensity of monsoon winds. The present productivity in the upwelling zone of the Arabian Sea may be as much as $\mathbf{3 0}$ times the amounts commonly observed in the oceans of the world (Romankevich, 1984). The historical changes in the intensity of monsoon winds are likely to have affected the distribution and quantity of or-

\footnotetext{
${ }^{1}$ Prell, W. L., Niitsuma, N., et al., 1991. Proc. ODP, Sci. Results, 117: College Station, TX (Ocean Drilling Program).

2 Department of Earth Sciences, Memorial University, St. John's, Newfoundland AlB 3X5, Canada.

${ }^{3}$ Department of Oceanography, University of British Columbia, Vancouver, British Columbia V6T IYl, Canada.

4 Present address: Department of Environmental Sciences, University of Virginia, Charlottesville, VA 22903, U.S.A.
}

ganic matter produced in upwelling regions. During interglacial periods, southwest monsoon winds are thought to have been strong, resulting in enhanced upwelling whereas upwelling tended to be weak during glacial events (Prell and Curry, 1981; Rossignol-Strick, 1983; Prell and van Campo, 1986; Prell and Kutzbach, 1987). The record of these variations may be preserved in the organic matter found in sediments beneath areas influenced by upwelling (Muller and Suess, 1979; Muller et al., 1983). Upwelling zones may vary spatially as well as temporally, complicating an interpretation of the organic sedimentary record (Reimers and Suess, 1983; ten Haven et al., 1989). The preservation of organic material in the sediments is also affected by hydrographic regime, particle size, oxidant content, and sedimentation rate (Emerson and Hedges, 1988; Calvert and Pedersen, in press).

The isotopic composition of organic matter in sediments has been used to suggest the influence of glacial episodes on marine sequences (Parker et al., 1972; Macko, 1989). To use stable isotope compositions to interpret input into the sedimentary record, the organic matter incorporated in sediments must reflect the original source carbon or nitrogen. Organic materials in the marine environment may originate in the photic zone, or in nearshore environments as terrigenous debris, or as a mixture of these two sources. This study uses organic carbon and nitrogen stable isotope compositions to delineate the origin of organic matter in sediments from the western Arabian Sea.

In numerous studies of marine environments, the relative contributions of terrigenous and marine inputs have been estimated from stable carbon isotope compositions. For example, in the Gulf of Mexico, surficial sediments contain increasing amounts of the heavier isotope of carbon $\left({ }^{13} \mathrm{C}\right)$ with increasing distance from land, and suggest a decreasing terrigenous carbon influence (Sackett and Thompson, 1963; Hedges and Parker, 1976; Gearing et al., 1977). In the deltas of both the Pedernales in Venezuela (Eckelmann et al., 1962) and Niger rivers (Gearing et al., 1977), woody fragments and more finely disseminated terrestrial plant debris give a clear terrigenous isotopic signature to deltaic sediments. In more northern Arctic environments, the transport of terrigenous material may be more extensive. In the Beaufort Sea, lateral transport of terrestrial debris is enhanced by ice-rafting (Gearing et al., 1977). These variations in source are recorded in the sedimentary record of a region. In the Gulf 


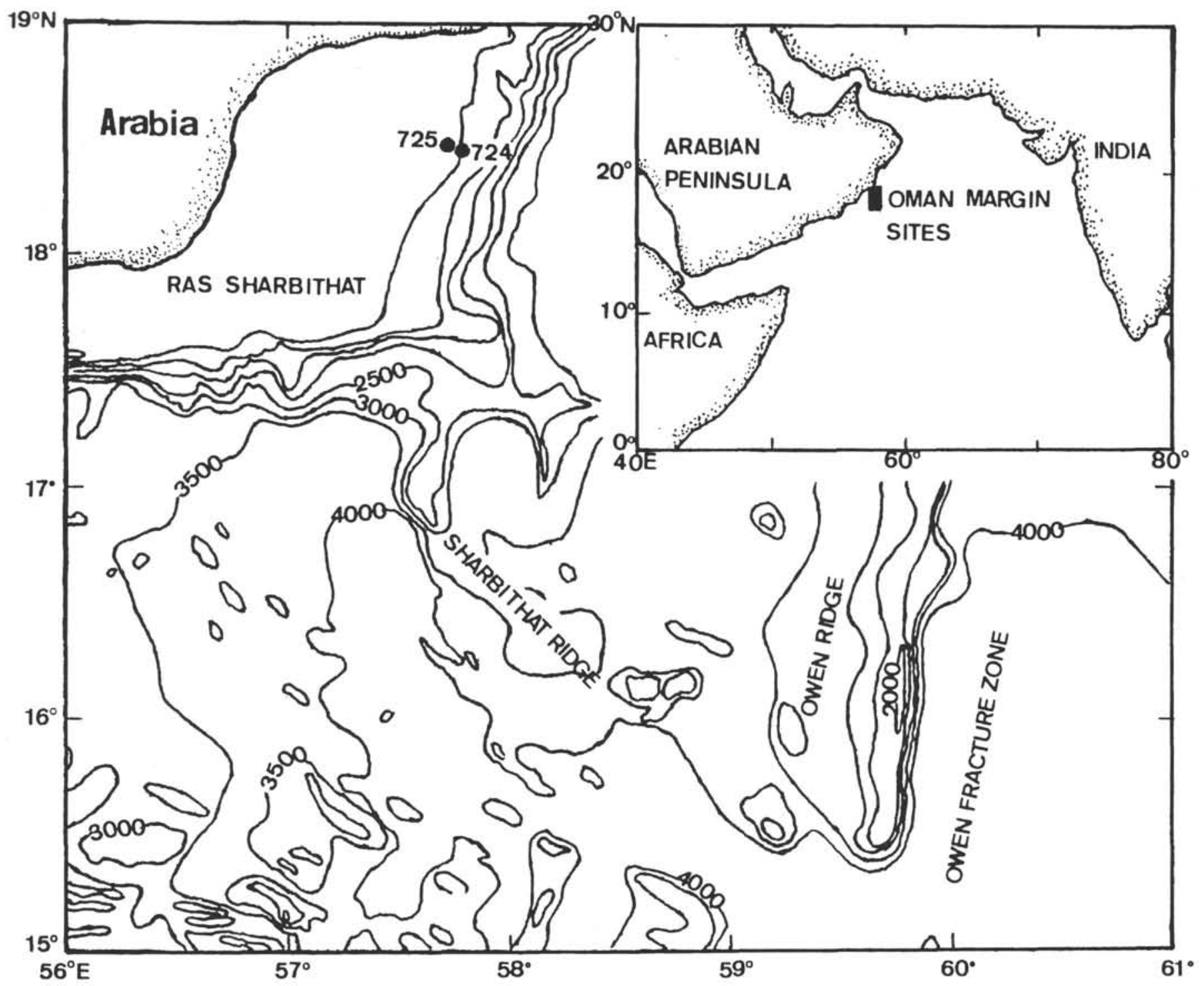

Figure 1. Map showing locations of Leg 117 sites in this study.

of Mexico, variations downcore have been correlated with glacial and interglacial episodes which are related to sea level lowering and the increasing influence of the Mississippi River on marine deposits (Parker et al., 1972; Newman et al., 1973). In environments which are dominated by marine productivity, other environmental parameters such as temperature, growth rate, species distribution, and $\mathrm{CO}_{2}$ availability may affect carbon isotopic compositions (Sackett et al., 1965; Degens et al., 1968; Gearing et al., 1977; Fontugne and Duplessy, 1978,; 1981; Degens, 1969). In Baffin Bay and the Labrador Sea (ODP Leg 105) and in the Weddell Sea (ODP Leg 113), large-scale changes in $\delta^{13} \mathrm{C}$ were interpreted to be strongly influenced by variations in marine planktonic productivity, and by the effect of ice-rafted debris on the overall accumulation of organic matter in sediments of the region (Macko, 1989; Macko and Pereira, 1990). Such variability would impact the organic isotopic record preserved in a sediment (Macko et al., 1987b). In this present study, it is less likely that certain of these variables will significantly impact the carbon isotopic compositions. For example, enhanced isotopic fractionation of carbon at low temperature and the strong influence of terrestrial debris are not expected owing to the warm waters and the lack of significant rivers in the region.
Temporal variation in river outflow and eolian inputs could have introduced measurable amounts of organic matter of terrigenous origin at specific times in the past, particularly during monsoon maxima.

Stable nitrogen isotopes are also useful indicators of the sources of organic material in marine sediments. Peters et al. (1978) used nitrogen isotope compositions to evaluate the relative contributions of terrigenous and marine inputs into a sediment. Because the major sources of nitrogen in terrestrial systems $\left(\mathrm{N}_{2}\right.$ via nitrogen fixation) are isotopically distinct from marine nitrogen (nitrate through nitrate reduction), these sources may be resolved in most environments. Further, in purely marine environments, the processes through which phytoplankton (or bacteria) incorporate nitrogen may be resolved if one can eliminate sources of nitrogen from land. The process of nitrogen fixation has a small isotopic fractionation associated with the utilization of molecular nitrogen. Such a process is easily distinguished from the more common mode of nitrate reduction in which phytoplankton fully utilize dissolved nitrate and reflect its isotopic signature (Macko et al., 1984). When algae are only able to use a portion of the dissolved nitrate, depleted ${ }^{15} \mathrm{~N}$ values may be evident, reflecting the isotopic fractionation associ- 
ated with that reduction (Macko et al., 1987a). Such changes may then be useful in the interpretation of the paleoceanographic record of an area. The organic matter in sediments deposited during periods of higher productivity, in which phytoplankton fully utilized the oceanic nitrate, may be more enriched in ${ }^{15} \mathrm{~N}$ than sediments associated with lower productivity in which larger fractionations occur.

However, the situation is complicated by factors which include microbial action, denitrification, diagenesis, and recycling of organics. Variations in isotopic signature may occur as a result of deamination reactions associated with organic rich materials (Wada, 1980; Zieman et al., 1984; Sigleo and Macko, 1985; Libes and Deuser, 1988). Such processes of alteration may be especially important in changing isotopic signatures below the euphotic zone (Altabet and McCarthy, 1986). Highly enriched isotopic compositions of nitrogen were observed in particulate material of the Indian Ocean; these enrichments were attributed to diagenetic effects in the water column (Saino and Hattori, 1980). With extensive denitrification of dissolved nitrate, the residual nitrate may become highly enriched in ${ }^{15} \mathrm{~N}$ (Cline and Kaplan, 1975; Liu, 1979). This remaining nitrate may serve as a nitrogen source for primary production, and if fully utilized, would be isotopically reflected in the newly produced organic matter.

\section{METHODS}

Sediment samples collected for isotope analysis were initially frozen. In this study, sampling frequency was typically five samples per $1 \mathrm{~m}$ interval, with the sample representing a homogenate of a $2 \mathrm{~cm}$ section of core. A large number of these samples thawed during shipment from the vessel to the laboratory (lower $180 \mathrm{~m}$ of Hole $724 \mathrm{C}$ and the samples from Site 725). These samples were refrozen upon receipt and maintained at $-10^{\circ} \mathrm{C}$ until processing. Samples were dried at $40^{\circ} \mathrm{C}$ and then acidified with $30 \% \mathrm{HCl}$ to remove carbonate. The carbonate-free residue was washed of all salts, because of the high carbonate content, and dried. A portion of the dried material was then weighed and combusted in quartz for $1 \mathrm{hr}$ at $850^{\circ} \mathrm{C}$ in the presence of purified cupric oxide wire and high purity granular copper (Macko et al., 1984). The $\mathrm{N}_{2}$ and $\mathrm{CO}_{2}$ gases obtained were cryogenically isolated from other combustion products and analyzed on a V.G. Micromass PRISM stable isotope ratio mass spectrometer. On the basis of replicate analyses of samples, the reproducibility in combustion and measurement is within $\pm 0.2 \%$. Isotope data are reported relative to standard materials as:

$$
\delta^{\mathrm{x}} \mathrm{E}=\left[\frac{\mathrm{R}_{\text {sample }}}{\mathrm{R}_{\text {standard }}}-1\right] \times 10^{3}
$$

where $\mathrm{X}$ is the isotope of the element $\mathrm{E}$ and $\mathrm{R}$ is the abundance ratio of the heavy to light isotope; the standard for ${ }^{15} \mathrm{~N}$ is atmospheric nitrogen and the standard for carbon is the Chicago PDB. For routine measurement, samples are analyzed vs. a laboratory secondary standard tank of either pure nitrogen or carbon dioxide gas. The organic carbon content was determined on the carbonate-free residue as volume of the carbon dioxide gas released in the combustion. The organic carbon percentages (Table 1) are not corrected for carbonate content of the sediment.

\section{RESULTS}

\section{Site 724}

At Site 724 , sampling was undertaken for high resolution analysis with 811 isolates being characterized from one hole (Hole 724C). This sampling allowed for resolution of fluctuations to be observed on the order of $1400-5700 \mathrm{yr}$ at the estimated sedimentation rate ranges of $142-35 \mathrm{~m} / \mathrm{m}$.y. The homog-
Table 1. Stable isotope organic geochemistry results.

\begin{tabular}{|c|c|c|c|c|}
\hline $\begin{array}{l}\text { Core and } \\
\text { section }\end{array}$ & $\begin{array}{l}\text { Depth } \\
\text { (mbsf) }\end{array}$ & $\delta^{15} \mathrm{~N}$ & $\delta^{13} \mathrm{C}$ & $\% \mathrm{C}$ \\
\hline \multicolumn{5}{|l|}{$117-724 C$ - } \\
\hline $1 \mathrm{H}-1$ & 0.22 & 8.1 & -20.0 & 6.1 \\
\hline $1 \mathrm{H}-1$ & 0.42 & 6.0 & -20.2 & 6.5 \\
\hline $1 \mathrm{H}-1$ & 0.62 & 8.1 & -19.6 & 2.5 \\
\hline $1 \mathrm{H}-1$ & 0.82 & 0.9 & -19.5 & 2.3 \\
\hline $1 \mathrm{H}-1$ & 1.02 & 7.3 & -19.5 & 1.4 \\
\hline $1 \mathrm{H}-1$ & 1.22 & 6.1 & -19.2 & 1.2 \\
\hline $1 \mathrm{H}-1$ & 1.24 & 5.5 & -19.1 & 0.8 \\
\hline $1 \mathrm{H}-2$ & 1.62 & 6.4 & -18.5 & 1.4 \\
\hline $1 \mathrm{H}-2$ & 1.82 & 6.0 & -19.3 & 1.7 \\
\hline $1 \mathrm{H}-2$ & 2.02 & 7.8 & -18.8 & 3.3 \\
\hline $1 \mathrm{H}-2$ & 2.22 & 6.5 & -19.0 & 2.7 \\
\hline $1 \mathrm{H}-2$ & 2.42 & 4.5 & -18.4 & 3.3 \\
\hline $1 \mathrm{H}-2$ & 2.58 & 10.1 & -19.0 & 2.6 \\
\hline $2 \mathrm{H}-1$ & 2.87 & 6.2 & -20.0 & 1.3 \\
\hline $2 \mathrm{H}-1$ & 3.02 & 5.9 & -20.8 & 1.2 \\
\hline $2 \mathrm{H}-1$ & 3.42 & 7.9 & -19.0 & 3.1 \\
\hline $2 \mathrm{H}-1$ & 3.62 & 5.6 & -19.3 & 1.1 \\
\hline $2 \mathrm{H}-1$ & 3.82 & 5.8 & -20.1 & 3.4 \\
\hline $2 \mathrm{H}-1$ & 4.02 & 10.1 & -20.0 & 4.9 \\
\hline $2 \mathrm{H}-1$ & 4.22 & 6.2 & -23.0 & 0.8 \\
\hline $2 \mathrm{H}-2$ & 4.42 & 6.4 & -21.7 & 1.0 \\
\hline $2 \mathrm{H}-2$ & 4.62 & 5.9 & -21.1 & 2.2 \\
\hline $2 \mathrm{H}-2$ & 5.02 & 6.9 & -20.2 & 4.3 \\
\hline $2 \mathrm{H}-2$ & 5.22 & 6.6 & -21.7 & 1.8 \\
\hline $2 \mathrm{H}-2$ & 5.42 & 6.6 & -21.9 & 1.4 \\
\hline $2 \mathrm{H}-2$ & 5.62 & 7.2 & -20.0 & 2.7 \\
\hline $2 \mathrm{H}-3$ & 5.82 & 6.5 & -20.6 & 0.7 \\
\hline $2 \mathrm{H}-3$ & 6.02 & 2.2 & -22.2 & 0.3 \\
\hline $2 \mathrm{H}-3$ & 6.22 & 6.0 & -19.4 & 1.1 \\
\hline $2 \mathrm{H}-3$ & 6.42 & 10.8 & -19.7 & 1.7 \\
\hline $2 \mathrm{H}-3$ & 6.62 & 11.0 & -19.3 & 1.6 \\
\hline $2 \mathrm{H}-3$ & 6.82 & 6.2 & -19.8 & 1.6 \\
\hline $2 \mathrm{H}-3$ & 7.02 & 4.7 & -19.6 & 2.1 \\
\hline $2 \mathrm{H}-3$ & 7.22 & 6.6 & -19.7 & 3.6 \\
\hline $2 \mathrm{H}-4$ & 7.42 & 10.7 & -19.5 & 2.3 \\
\hline $2 \mathrm{H}-4$ & 7.62 & 8.4 & -19.4 & 4.0 \\
\hline $2 \mathrm{H}-4$ & 7.82 & 9.4 & -19.9 & 5.3 \\
\hline $2 \mathrm{H}-4$ & 8.02 & 8.3 & -19.8 & 2.4 \\
\hline $2 \mathrm{H}-4$ & 8.22 & 9.0 & -19.9 & 3.4 \\
\hline $2 \mathrm{H}-4$ & 8.42 & 8.1 & -19.9 & 3.6 \\
\hline $2 \mathrm{H}-4$ & 8.62 & 10.0 & -19.5 & 4.5 \\
\hline $2 \mathrm{H}-5$ & 8.82 & 9.4 & -20.1 & 3.4 \\
\hline $2 \mathrm{H}-5$ & 9.02 & 10.7 & -19.2 & 2.8 \\
\hline $2 \mathrm{H}-5$ & 9.22 & 7.0 & -20.6 & 3.9 \\
\hline $2 \mathrm{H}-5$ & 9.42 & 11.0 & -20.0 & 1.6 \\
\hline $2 \mathrm{H}-5$ & 9.62 & 5.6 & -21.0 & 2.0 \\
\hline $2 \mathrm{H}-5$ & 9.82 & 10.4 & -20.3 & 2.8 \\
\hline $2 \mathrm{H}-5$ & 10.02 & 7.8 & -19.6 & 1.1 \\
\hline $2 \mathrm{H}-5$ & 10.22 & 15.9 & -19.8 & 0.8 \\
\hline $2 \mathrm{H}-6$ & 10.42 & 6.6 & -20.1 & 1.1 \\
\hline $2 \mathrm{H}-6$ & 10.62 & 10.5 & -19.6 & 0.8 \\
\hline $2 \mathrm{H}-6$ & 10.82 & 11.1 & -19.6 & 1.4 \\
\hline $2 \mathrm{H}-6$ & 11.02 & 9.4 & -19.5 & 1.4 \\
\hline $2 \mathrm{H}-6$ & 11.22 & 11.5 & -20.0 & 1.5 \\
\hline $2 \mathrm{H}-6$ & 11.42 & 6.6 & -19.9 & 1.7 \\
\hline $2 \mathrm{H}-6$ & 11.62 & 8.7 & -20.7 & 4.1 \\
\hline $2 \mathrm{H}-7$ & 11.82 & 12.2 & -19.5 & 2.9 \\
\hline $2 \mathrm{H}-7$ & 12.02 & 7.9 & -20.8 & 1.9 \\
\hline $2 \mathrm{H}-7$ & 12.22 & 5.5 & -19.7 & 1.5 \\
\hline $2 \mathrm{H}-7$ & 12.42 & 9.2 & -19.4 & 2.2 \\
\hline $3 \mathrm{H}-1$ & 12.42 & 8.8 & -19.6 & 3.3 \\
\hline $3 \mathrm{H}-1$ & 12.62 & 4.9 & -19.5 & 2.6 \\
\hline $3 \mathrm{H}-1$ & 12.82 & 6.6 & -19.5 & 1.8 \\
\hline $3 \mathrm{H}-1$ & 13.02 & 12.5 & -19.5 & 2.1 \\
\hline $3 \mathrm{H}-1$ & 13.22 & 8.0 & -20.1 & 4.6 \\
\hline $3 \mathrm{H}-1$ & 13.42 & 8.3 & -19.5 & 1.5 \\
\hline $3 \mathrm{H}-1$ & 13.62 & 4.4 & -19.5 & 1.9 \\
\hline $3 \mathrm{H}-2$ & 13.82 & 5.8 & -19.8 & 1.9 \\
\hline $3 \mathrm{H}-2$ & 14.02 & 6.1 & -20.0 & 2.8 \\
\hline $3 \mathrm{H}-2$ & 14.22 & 6.6 & -19.9 & 0.8 \\
\hline $3 \mathrm{H}-2$ & 14.42 & 12.5 & -19.4 & 1.3 \\
\hline $3 \mathrm{H}-2$ & 14.62 & 13.2 & -19.8 & 1.2 \\
\hline $3 \mathrm{H}-2$ & 14.82 & 10.1 & -19.8 & 1.3 \\
\hline $3 \mathrm{H}-2$ & 15.02 & 10.2 & -19.6 & 1.5 \\
\hline $3 \mathrm{H}-3$ & 15.22 & 9.2 & -19.5 & 1.9 \\
\hline
\end{tabular}


Table 1 (continued).

\begin{tabular}{|c|c|c|c|c|}
\hline $\begin{array}{l}\text { Core and } \\
\text { section }\end{array}$ & $\begin{array}{l}\text { Depth } \\
\text { (mbsf) }\end{array}$ & $\delta^{15} \mathrm{~N}$ & $\delta^{13} \mathrm{C}$ & $\% \mathrm{C}$ \\
\hline \multicolumn{5}{|c|}{ 117-724C-(Cont.) } \\
\hline $3 \mathrm{H}-3$ & 15.42 & 11.3 & -19.5 & 1.7 \\
\hline $3 \mathrm{H}-3$ & 15.62 & 5.4 & -19.8 & 2.9 \\
\hline $3 \mathrm{H}-3$ & 15.82 & 11.2 & -19.3 & 1.9 \\
\hline $3 \mathrm{H}-3$ & 16.22 & 9.7 & -21.4 & 5.2 \\
\hline $3 \mathrm{H}-3$ & 16.42 & 5.2 & -19.4 & 2.3 \\
\hline $3 \mathrm{H}-3$ & 16.62 & 15.1 & -20.0 & 0.9 \\
\hline $3 \mathrm{H}-4$ & 16.82 & 11.5 & -19.5 & 1.3 \\
\hline $3 \mathrm{H}-4$ & 17.02 & 12.3 & -19.6 & 1.6 \\
\hline $3 \mathrm{H}-4$ & 17.22 & 10.0 & -20.1 & 2.5 \\
\hline $3 \mathrm{H}-4$ & 17.42 & 8.9 & -21.3 & 1.6 \\
\hline $3 \mathrm{H}-4$ & 17.62 & 5.5 & -19.8 & 1.5 \\
\hline $3 \mathrm{H}-4$ & 17.82 & 10.4 & -19.7 & 1.7 \\
\hline $3 \mathrm{H}-4$ & 18.02 & 7.6 & -19.0 & 2.2 \\
\hline $3 \mathrm{H}-5$ & 18.22 & 5.8 & -19.5 & 2.2 \\
\hline $3 \mathrm{H}-5$ & 18.42 & 7.7 & -20.0 & 2.9 \\
\hline $3 \mathrm{H}-5$ & 18.62 & 4.3 & -19.6 & 2.3 \\
\hline $3 \mathrm{H}-5$ & 18.82 & 9.4 & -19.7 & 2.0 \\
\hline $3 \mathrm{H}-5$ & 19.02 & 12.6 & -19.5 & 1.7 \\
\hline $3 \mathrm{H}-5$ & 19.22 & 9.4 & -19.6 & 1.9 \\
\hline $3 \mathrm{H}-5$ & 19.42 & 5.6 & -19.8 & 1.9 \\
\hline $3 \mathrm{H}-5$ & 19.62 & 9.4 & -19.6 & 1.9 \\
\hline $3 \mathrm{H}-6$ & 19.82 & 12.4 & -20.1 & 2.7 \\
\hline $3 \mathrm{H}-6$ & 20.02 & 8.6 & -19.8 & 3.6 \\
\hline $3 \mathrm{H}-6$ & 20.22 & 9,8 & -20.8 & 2.7 \\
\hline $3 \mathrm{H}-6$ & 20.42 & 10.6 & -19.6 & 3.5 \\
\hline $3 \mathrm{H}-6$ & 20.62 & 10.6 & -20.5 & 3.6 \\
\hline $3 \mathrm{H}-6$ & 20.82 & 12.6 & -20.0 & 4.0 \\
\hline $3 \mathrm{H}-6$ & 21.02 & 9.1 & -19.4 & 2.1 \\
\hline $3 \mathrm{H}-7$ & 21.22 & 7.6 & -19.6 & 1.9 \\
\hline $3 \mathrm{H}-7$ & 21.42 & 2.2 & -19.9 & 2.5 \\
\hline $3 \mathrm{H}-7$ & 21.62 & 9.9 & -19.5 & 2.8 \\
\hline $3 \mathrm{H}-7$ & 21.82 & 8.7 & -20.5 & 3.7 \\
\hline $4 \mathrm{H}-1$ & 21.80 & 12.8 & -20.4 & 1.6 \\
\hline $4 \mathrm{H}-1$ & 21.92 & 9.7 & -20.5 & 2.2 \\
\hline $4 \mathrm{H}-1$ & 22.12 & 11.9 & -20.2 & 1.7 \\
\hline $4 \mathrm{H}-1$ & 22.32 & 12.3 & -19.9 & 1.2 \\
\hline $4 \mathrm{H}-1$ & 22.52 & 6.1 & -20.1 & 2.6 \\
\hline $4 \mathrm{H}-1$ & 22.72 & 10.4 & -20.1 & 3.6 \\
\hline $4 \mathrm{H}-1$ & 22.92 & 9.0 & -20.1 & 4.1 \\
\hline $4 \mathrm{H}-1$ & 23.12 & 10.8 & -19.5 & 2.1 \\
\hline $4 \mathrm{H}-2$ & 23.32 & 11.4 & -20.3 & 3.1 \\
\hline $4 \mathrm{H}-2$ & 23.52 & 11.2 & -20.1 & 3.7 \\
\hline $4 \mathrm{H}-2$ & 23.72 & 10.7 & -20.0 & 2.3 \\
\hline $4 \mathrm{H}-2$ & 23.92 & 10.9 & -19.5 & 2.9 \\
\hline $4 \mathrm{H}-2$ & 24.12 & 9.3 & -19.4 & 2.7 \\
\hline $4 \mathrm{H}-2$ & 24.32 & 8.6 & -21.1 & 1.5 \\
\hline $4 \mathrm{H}-2$ & 24.52 & 8.8 & -20.2 & 1.6 \\
\hline $4 \mathrm{H}-3$ & 24.72 & 5.2 & -21.1 & 1.0 \\
\hline $4 \mathrm{H}-3$ & 24.92 & 14.0 & -22.6 & 0.4 \\
\hline $4 \mathrm{H}-3$ & 25.12 & 10.7 & -19.2 & 1.2 \\
\hline $4 \mathrm{H}-3$ & 25.32 & 9.6 & -21.4 & 0.3 \\
\hline $4 \mathrm{H}-3$ & 25.52 & 10.5 & -20.1 & 1.6 \\
\hline $4 \mathrm{H}-3$ & 25.72 & 12.4 & -19.6 & 2.4 \\
\hline $4 \mathrm{H}-3$ & 25.92 & 11.3 & -19.8 & 2.6 \\
\hline $4 \mathrm{H}-3$ & 26.12 & 9.8 & -19.4 & 3.3 \\
\hline $4 \mathrm{H}-4$ & 26.32 & 8.7 & -20.4 & 2.6 \\
\hline $4 \mathrm{H}-4$ & 26.52 & 9.1 & -20.0 & 5.8 \\
\hline $4 \mathrm{H}-4$ & 26.72 & 9.1 & -19.8 & 3.3 \\
\hline $4 \mathrm{H}-4$ & 26.92 & 8.4 & -20.0 & 2.8 \\
\hline $4 \mathrm{H}-4$ & 27.12 & 10.0 & -19.6 & 2.4 \\
\hline $4 \mathrm{H}-4$ & 27.32 & 4.6 & -20.0 & 3.3 \\
\hline $4 \mathrm{H}-4$ & 27.52 & 6.5 & -21.3 & 3.3 \\
\hline $4 \mathrm{H}-5$ & 27.72 & 7.9 & -21.1 & 5.0 \\
\hline $4 \mathrm{H}-5$ & 27.92 & 6.5 & -20.7 & 6.1 \\
\hline 4H-5 & 28.12 & 10.9 & -20.5 & 2.4 \\
\hline $4 \mathrm{H}-5$ & 28.32 & 4.1 & -20.3 & 0.1 \\
\hline $4 \mathrm{H}-5$ & 28.52 & 7.2 & -20.5 & 4.2 \\
\hline $4 \mathrm{H}-5$ & 28.72 & 14.0 & -20.1 & 0.8 \\
\hline $4 \mathrm{H}-5$ & 28.92 & 11.8 & -21.5 & 0.9 \\
\hline $4 \mathrm{H}-5$ & 29.12 & 10.3 & -22.2 & 0.8 \\
\hline $4 \mathrm{H}-6$ & 29.32 & 9.2 & -21.0 & 0.8 \\
\hline $4 \mathrm{H}-6$ & 29.52 & 12.5 & -24.7 & 0.2 \\
\hline $4 \mathrm{H}-6$ & 29.72 & 16.0 & -21.0 & 0.5 \\
\hline $4 \mathrm{H}-6$ & 29.92 & 13.0 & -23.6 & 0.9 \\
\hline $4 \mathrm{H}-6$ & 30.12 & 9.3 & -23.5 & 1.3 \\
\hline $4 \mathrm{H}-6$ & 30.32 & 7.8 & -20.0 & 2.2 \\
\hline $4 \mathrm{H}-6$ & 30.52 & 9.9 & -20.2 & 1.9 \\
\hline
\end{tabular}

Table 1 (continued).

\begin{tabular}{|c|c|c|c|c|}
\hline $\begin{array}{l}\text { Core and } \\
\text { section }\end{array}$ & $\begin{array}{l}\text { Depth } \\
\text { (mbsf) }\end{array}$ & $\delta^{15} \mathrm{~N}$ & ${ }^{\delta^{13} \mathrm{C}}$ & $\% \mathrm{C}$ \\
\hline \multicolumn{5}{|c|}{ 117-724C-(Cont.) } \\
\hline $4 \mathrm{H}-7$ & 30.88 & 11.4 & -20.1 & 2.2 \\
\hline $4 \mathrm{H}-7$ & 31.08 & 10.7 & -19.8 & 2.0 \\
\hline $4 \mathrm{H}-7$ & 31.28 & 9.2 & -19.7 & 2.2 \\
\hline $4 \mathrm{H}-7$ & 31.48 & 6.0 & -20.1 & 2.3 \\
\hline $5 \mathrm{H}-1$ & 31.28 & 5.4 & -20.0 & 2.3 \\
\hline $5 \mathrm{H}-1$ & 31.42 & 9.5 & -20.1 & 1.5 \\
\hline $5 \mathrm{H}-1$ & 31.62 & 11.2 & -20.2 & 1.5 \\
\hline $5 \mathrm{H}-1$ & 32.02 & 11.3 & -20.2 & 2.0 \\
\hline $5 \mathrm{H}-1$ & 32.22 & 8.0 & -20.1 & 2.1 \\
\hline $5 \mathrm{H}-1$ & 32.42 & 5.6 & -20.7 & 2.2 \\
\hline $5 \mathrm{H}-1$ & 32.62 & 7.9 & -20.3 & 3.5 \\
\hline $5 \mathrm{H}-2$ & 32.82 & 11.9 & -20.3 & 2.2 \\
\hline $5 \mathrm{H}-2$ & 33.02 & 11.1 & -20.8 & 1.6 \\
\hline $5 \mathrm{H}-2$ & 33.42 & 11.8 & -21.0 & 0.8 \\
\hline $5 \mathrm{H}-2$ & 33.62 & 10.2 & -20.6 & 0.6 \\
\hline $5 \mathrm{H}-2$ & 33.82 & 14.3 & -19.5 & 1.6 \\
\hline $5 \mathrm{H}-2$ & 34.02 & 8.5 & -19.8 & 1.7 \\
\hline $5 \mathrm{H}-3$ & 34.42 & 15.5 & -19.8 & 1.3 \\
\hline $5 \mathrm{H}-3$ & 34.62 & 14.1 & -20.1 & 1.0 \\
\hline $5 \mathrm{H}-3$ & 34.82 & 5.3 & -19.4 & 1.1 \\
\hline $5 \mathrm{H}-3$ & 35.02 & 10.5 & -19.9 & 0.9 \\
\hline $5 \mathrm{H}-3$ & 35.22 & 11.9 & -18.5 & 2.3 \\
\hline $5 \mathrm{H}-3$ & 35.42 & 10.0 & -19.8 & 2.4 \\
\hline $5 \mathrm{H}-3$ & 35.46 & 14.2 & -19.3 & 2.0 \\
\hline $5 \mathrm{H}-4$ & 35.48 & 5.8 & -19.5 & 2.2 \\
\hline $5 \mathrm{H}-4$ & 36.02 & 7.2 & -19.6 & 3.0 \\
\hline $5 \mathrm{H}-4$ & 36.22 & 12.5 & -18.8 & 4.2 \\
\hline $5 \mathrm{H}-4$ & 36.42 & 6.8 & -20.1 & 4.2 \\
\hline $5 \mathrm{H}-4$ & 36.62 & 7.9 & -19.7 & 3.7 \\
\hline $5 \mathrm{H}-4$ & 36.82 & 7.1 & -19.4 & 3.0 \\
\hline $5 \mathrm{H}-4$ & 37.02 & 8.5 & -19.6 & 4.5 \\
\hline $5 \mathrm{H}-5$ & 37.22 & 9.6 & -18.5 & 2.6 \\
\hline $5 \mathrm{H}-5$ & 37.42 & 11.0 & -19.5 & 3.5 \\
\hline $5 \mathrm{H}-5$ & 37.62 & 6.8 & -20.2 & 3.2 \\
\hline $5 \mathrm{H}-5$ & 38.02 & 12.6 & -19.6 & 3.3 \\
\hline $5 \mathrm{H}-5$ & 38.22 & 11.8 & -19.9 & 3.4 \\
\hline $5 \mathrm{H}-5$ & 38.42 & 10.4 & -19.6 & 3.8 \\
\hline $5 \mathrm{H}-5$ & 38.62 & 9.8 & -19.9 & 3.0 \\
\hline $5 \mathrm{H}-6$ & 38.82 & 15.3 & -19.9 & 3.0 \\
\hline $5 \mathrm{H}-6$ & 39.02 & 13.8 & -20.0 & 3.8 \\
\hline $5 \mathrm{H}-6$ & 39.22 & 12.9 & -18.8 & 3.4 \\
\hline $5 \mathrm{H}-6$ & 39.42 & 11.9 & -21.9 & 1.9 \\
\hline $5 \mathrm{H}-6$ & 39.62 & 13.2 & -23.3 & 2.4 \\
\hline $5 \mathrm{H}-6$ & 39.82 & 12.1 & -20.3 & 2.4 \\
\hline $5 \mathrm{H}-6$ & 40.02 & 11.9 & -19.4 & 2.1 \\
\hline $5 \mathrm{H}-7$ & 40.22 & 9.3 & -19.9 & 3.0 \\
\hline $5 \mathrm{H}-7$ & 40.42 & 12.3 & -20.0 & 1.7 \\
\hline $5 \mathrm{H}-7$ & 40.62 & 11.0 & -19.6 & 1.6 \\
\hline $5 \mathrm{H}-7$ & 40.82 & 11.7 & -19.6 & 2.1 \\
\hline $6 \mathrm{X}-1$ & 40.84 & 11.7 & -20.8 & 3.2 \\
\hline $6 \mathrm{X}-1$ & 41.02 & 9.7 & -21.4 & 3.4 \\
\hline $6 \mathrm{X}-1$ & 41.22 & 9.4 & -20.4 & 5.1 \\
\hline $6 \mathrm{X}-1$ & 41.42 & 9.6 & -19.9 & 3.6 \\
\hline $6 \mathrm{X}-1$ & 41.62 & 11.1 & -19.8 & 2.8 \\
\hline $6 \mathrm{X}-1$ & 41.82 & 11.1 & -19.5 & 2.2 \\
\hline $6 \mathrm{X}-1$ & 42.02 & 9.6 & -19.9 & 2.7 \\
\hline $6 \mathrm{X}-1$ & 42.22 & 9.8 & -19.9 & 4.0 \\
\hline $6 \times-2$ & 42.42 & 13.0 & -22.6 & 1.8 \\
\hline $6 \mathrm{X}-2$ & 42.62 & 8.7 & -20.7 & 5.1 \\
\hline $6 \mathrm{X}-2$ & 42.82 & 11.5 & -20.6 & 4.2 \\
\hline $6 \mathrm{X}-2$ & 43.02 & 10.0 & -21.5 & 1.3 \\
\hline $6 \mathrm{X}-2$ & 43.22 & 8.5 & -20.3 & 1.2 \\
\hline $6 \mathrm{X}-2$ & 43.42 & 9.9 & -19.9 & 2.5 \\
\hline $6 \mathrm{X}-2$ & 43.62 & 11.3 & -20.3 & 2.3 \\
\hline $6 \mathrm{X}-3$ & 43.82 & 11.6 & -20.0 & 1.9 \\
\hline $6 \mathrm{X}-3$ & 44.02 & 14.7 & -20.1 & 1.9 \\
\hline $6 \mathrm{X}-3$ & 44.22 & 7.4 & -19.2 & 2.3 \\
\hline $6 \mathrm{X}-3$ & 44.42 & 11.2 & -20.3 & 1.7 \\
\hline $6 \mathrm{X}-3$ & 44.62 & 8.1 & -19.8 & 2.9 \\
\hline $6 \mathrm{X}-3$ & 44.82 & 9.8 & -19.4 & 2.6 \\
\hline $6 \mathrm{X}-3$ & 45.02 & 11.5 & -19.7 & 2.1 \\
\hline $6 X-3$ & 45.22 & 11.1 & -18.9 & 2.0 \\
\hline $6 \times-4$ & 45.42 & 12.2 & -20.3 & 1.3 \\
\hline $6 \times-4$ & 45.62 & 9.7 & -19.6 & 3.5 \\
\hline $6 \times-4$ & 45.82 & 8.2 & -19.4 & \\
\hline $6 \times-4$ & 46.02 & 10.8 & -19.8 & \\
\hline
\end{tabular}


Table 1 (continued).

\begin{tabular}{|c|c|c|c|c|}
\hline $\begin{array}{l}\text { Core and } \\
\text { section }\end{array}$ & $\begin{array}{l}\text { Depth } \\
\text { (mbsf) }\end{array}$ & $\delta^{15} \mathrm{~N}$ & $\delta^{13} \mathrm{C}$ & $\% \mathrm{C}$ \\
\hline \multicolumn{5}{|c|}{ 117-724C-(Cont.) } \\
\hline $6 \times-4$ & 46.22 & 10.9 & -19.6 & 4.3 \\
\hline $6 \times-4$ & 46.42 & 5.4 & -19.7 & 3.1 \\
\hline $6 \times-4$ & 46.62 & 8.4 & -20.0 & 2.5 \\
\hline $6 \times-5$ & 46.82 & 9.7 & -20.1 & 2.4 \\
\hline $6 X-5$ & 47.02 & 7.1 & -20.2 & 2.6 \\
\hline $6 X-5$ & 47.22 & 11.3 & -20.7 & 3.4 \\
\hline $6 \times-5$ & 47.42 & 12.5 & -20.0 & 1.8 \\
\hline $6 X-5$ & 47.62 & 7.7 & -20.2 & 2.3 \\
\hline $6 \times-5$ & 47.82 & 12.4 & -19.8 & 1.6 \\
\hline $6 X-5$ & 48.02 & 11.2 & -20.6 & 1.7 \\
\hline $6 \times-5$ & 48.22 & 12.0 & -19.2 & 1.6 \\
\hline $6 \times-6$ & 48.42 & 6.1 & -20.6 & 2.6 \\
\hline $6 \mathrm{X}-6$ & 48.62 & 10.2 & -19.6 & 2.2 \\
\hline $6 \times-6$ & 48.82 & 10.8 & -21.2 & 4.0 \\
\hline $6 \mathrm{X}-6$ & 49.02 & 9.3 & -19.9 & 1.8 \\
\hline $6 \times-6$ & 49.22 & 10.3 & -19.2 & 1.2 \\
\hline $7 X-1$ & 50.72 & 12.5 & -21.0 & 6.2 \\
\hline $7 X-1$ & 50.92 & 9.8 & -20.9 & 5.2 \\
\hline $7 X-1$ & 51.12 & 10.0 & -20.4 & 2.5 \\
\hline $7 X-1$ & 51.32 & 11.1 & -20.9 & 5.2 \\
\hline $7 X-1$ & 51.52 & 10.3 & -20.8 & 3.6 \\
\hline $7 X-1$ & 51.72 & 5.5 & -20.2 & 2.2 \\
\hline $7 X-1$ & 51.92 & 8.0 & -20.1 & 1.4 \\
\hline $7 X-2$ & 52.12 & 9.6 & -20.2 & 1.7 \\
\hline $7 X-2$ & 52.32 & 10.0 & -20.2 & 2.0 \\
\hline $7 X-2$ & 52.52 & 4.5 & -20.5 & 1.6 \\
\hline $7 X-2$ & 52.72 & 8.1 & -19.9 & 1.2 \\
\hline $7 X-2$ & 52.92 & 4.5 & -19.0 & 1.0 \\
\hline $7 X-2$ & 53.12 & 16.9 & -20.0 & 1.6 \\
\hline $7 X-2$ & 53.32 & 12.0 & -20.6 & 1.1 \\
\hline $7 X-3$ & 53.52 & 8.1 & -19.5 & 0.8 \\
\hline $7 X-3$ & 53.72 & 13.1 & -20.2 & 1.0 \\
\hline $7 X-3$ & 53.92 & 10.2 & -20.6 & 1.3 \\
\hline $7 X-3$ & 54.32 & 12.6 & -20.2 & 1.8 \\
\hline $7 X-3$ & 54.52 & 7.8 & -20.2 & 2.1 \\
\hline $7 X-3$ & 54.72 & 6.1 & -20.1 & 3.0 \\
\hline $7 X-3$ & 54.92 & 9.9 & -19.8 & 3.1 \\
\hline $7 X-4$ & 55.12 & 6.9 & -21.2 & 3.8 \\
\hline $7 X-4$ & 55.32 & 11.9 & -20.4 & 4.4 \\
\hline $7 X-4$ & 55.52 & 11.0 & -20.1 & 5.1 \\
\hline $7 \times-4$ & 55.72 & 11.9 & -20.8 & 4.3 \\
\hline $7 X-4$ & 55.92 & 9.7 & -22.9 & 2.5 \\
\hline $7 \times-4$ & 56.12 & 5.7 & -19.4 & 2.8 \\
\hline $7 X-4$ & 56.32 & 8.6 & -21.5 & 4.6 \\
\hline $7 X-5$ & 56.72 & 6.7 & -20.4 & 2.9 \\
\hline $7 X-5$ & 56.92 & 7.5 & -20.3 & 3.3 \\
\hline $7 X-5$ & 57.12 & 9.1 & -20.6 & 3.7 \\
\hline $7 X-5$ & 57.32 & 11.3 & -20.6 & 2.2 \\
\hline $7 X-5$ & 57.52 & 9.3 & -17.7 & 1.9 \\
\hline $7 X-5$ & 57.72 & 10.1 & -20.5 & 1.9 \\
\hline $7 X-5$ & 57.92 & 8.3 & -19.8 & 2.1 \\
\hline $7 X-6$ & 58.12 & 9.0 & -20.3 & 2.7 \\
\hline $7 X-6$ & 58.32 & 12.3 & -19.3 & 2.8 \\
\hline $7 X-6$ & 58.52 & 8.2 & -20.1 & 2.6 \\
\hline $7 X-6$ & 58.72 & 8.9 & -20.1 & 3.5 \\
\hline $8 X-1$ & 60.22 & 18.9 & -20.2 & 4.1 \\
\hline $8 X-1$ & 60.42 & 6.2 & -21.1 & 4.1 \\
\hline $8 X-1$ & 60.62 & 6.7 & -20.7 & 4.9 \\
\hline $8 \times-1$ & 60.82 & 5.7 & -20.4 & 3.6 \\
\hline $8 X-1$ & 61.02 & 5.4 & -20.5 & 4.7 \\
\hline $8 X-1$ & 61.22 & 8.2 & -20.3 & 3.3 \\
\hline $8 X-1$ & 61.42 & 8.7 & -20.2 & 3.3 \\
\hline $8 X-1$ & 61.62 & 6.1 & -20.9 & 4.7 \\
\hline $8 X-2$ & 61.82 & 8.8 & -19.9 & 5.7 \\
\hline $8 X-2$ & 62.02 & 8.9 & -20.1 & 3.5 \\
\hline $8 X-2$ & 62.22 & 4.7 & -20.5 & 3.6 \\
\hline $8 X-2$ & 62.42 & 6.4 & -21.0 & 5.2 \\
\hline $8 X-2$ & 62.62 & 16.4 & -20.5 & 4.5 \\
\hline $8 X-2$ & 62.82 & 5.8 & -20.7 & 4.7 \\
\hline $8 X-2$ & 63.02 & 4.9 & -19.0 & 1.4 \\
\hline $8 X-3$ & 63.22 & 4.4 & -19.3 & 1.1 \\
\hline $8 X-3$ & 63.42 & 6.3 & -19.2 & 1.3 \\
\hline $8 X-3$ & 63.62 & 10.7 & -18.9 & 1.1 \\
\hline $8 X-3$ & 63.82 & 11.0 & -20.1 & 2.3 \\
\hline $8 X-3$ & 64.02 & 12.9 & -19.5 & 1.2 \\
\hline $8 X-3$ & 64.22 & 11.2 & -19.5 & 1.5 \\
\hline
\end{tabular}

Table 1 (continued).

\begin{tabular}{|c|c|c|c|c|}
\hline $\begin{array}{l}\text { Core and } \\
\text { section }\end{array}$ & $\begin{array}{l}\text { Depth } \\
\text { (mbsf) }\end{array}$ & $\delta^{15} \mathrm{~N}$ & $\delta^{13} \mathrm{C}$ & $\% \mathrm{C}$ \\
\hline \multicolumn{5}{|c|}{ 117-724C-(Cont.) } \\
\hline $8 X-3$ & 64.42 & 6.2 & -19.6 & 1.8 \\
\hline $8 X-3$ & 64.62 & 11.2 & -20.3 & 4.3 \\
\hline $8 X-4$ & 64.82 & 4.3 & -19.7 & 4.0 \\
\hline $8 X-4$ & 65.02 & 4.7 & -20.4 & 2.8 \\
\hline $8 X-4$ & 65.22 & 6.8 & -20.3 & 3.8 \\
\hline $8 X-4$ & 65.42 & 8.3 & -19.4 & 3.1 \\
\hline $8 X-4$ & 65.62 & 7.1 & -20.7 & 5.1 \\
\hline $8 X-4$ & 65.82 & 7.0 & -21.2 & 5.9 \\
\hline $8 X-4$ & 66.02 & 5.7 & -21.7 & 6.5 \\
\hline $8 X-5$ & 66.22 & 7.0 & -23.5 & 12.9 \\
\hline $8 X-5$ & 66.42 & 9.0 & -21.2 & 6.5 \\
\hline $8 X-5$ & 66.62 & 11.4 & -20.5 & 6.0 \\
\hline $8 X-5$ & 66.82 & 10.4 & -20.9 & 6.0 \\
\hline $8 X-5$ & 67.02 & 12.6 & -20.0 & 2.5 \\
\hline $8 X-5$ & 67.22 & 12.6 & -21.0 & 1.2 \\
\hline $8 X-5$ & 67.42 & 6.1 & -20.1 & 1.7 \\
\hline $8 X-5$ & 67.62 & 14.0 & -18.7 & 1.6 \\
\hline $8 X-6$ & 67.82 & 11.6 & -22.0 & 2.9 \\
\hline $8 X-6$ & 68.02 & 4.6 & -20.2 & 3.8 \\
\hline $8 X-6$ & 68.22 & 7.5 & -19.9 & 3.9 \\
\hline $9 X-1$ & 70.02 & 12.5 & -20.1 & 4.0 \\
\hline $9 X-1$ & 70.22 & 12.4 & -19.8 & 4.4 \\
\hline $9 X-1$ & 70.42 & 8.2 & -19.9 & 3.0 \\
\hline $9 X-1$ & 70.62 & 2.8 & -21.3 & 1.3 \\
\hline $9 X-1$ & 70.82 & 6.5 & -20.5 & 3.6 \\
\hline $9 X-1$ & 71.02 & 9.5 & -19.9 & 5.0 \\
\hline $9 X-1$ & 71.22 & 8.3 & -20.4 & 4.4 \\
\hline $9 X-2$ & 71.42 & 7.7 & -20.8 & 3.9 \\
\hline $9 X-2$ & 71.62 & 8.9 & -20.2 & 2.0 \\
\hline $9 X-2$ & 71.82 & 6.0 & -21.4 & 3.3 \\
\hline $9 X-2$ & 72.02 & 13.0 & -19.8 & 4.4 \\
\hline $9 X-2$ & 72.22 & 12.0 & -19.9 & 3.3 \\
\hline $9 X-2$ & 72.42 & 5.4 & -21.0 & 3.4 \\
\hline $9 X-2$ & 72.62 & 8.5 & -21.6 & 2.4 \\
\hline $9 X-3$ & 72.82 & 12.6 & -20.4 & 4.3 \\
\hline $9 X-3$ & 73.02 & 7.1 & -19.9 & 3.8 \\
\hline $9 X-3$ & 73.42 & 4.1 & -20.4 & 3.4 \\
\hline $9 X-3$ & 73.62 & 7.6 & -20.2 & 4.0 \\
\hline $9 X-3$ & 73.82 & 6.9 & -21.9 & 5.2 \\
\hline $9 X-3$ & 74.02 & 10.4 & -22.0 & 6.4 \\
\hline $9 X-3$ & 74.22 & 11.3 & -21.2 & 5.4 \\
\hline $9 X-3$ & 74.42 & 4.7 & -21.8 & 8.8 \\
\hline $9 X-4$ & 74.62 & 12.2 & -21.2 & 6.8 \\
\hline $9 X-4$ & 74.82 & 7.1 & -23.0 & 3.6 \\
\hline $9 X-4$ & 75.02 & 9.9 & -21.5 & 4.3 \\
\hline $9 X-4$ & 75.22 & 9.8 & -20.8 & 4.8 \\
\hline $9 \times-4$ & 75.42 & 11.9 & -20.7 & 5.0 \\
\hline $9 X-5$ & 75.82 & 10.8 & -19.6 & 3.1 \\
\hline $9 X-5$ & 76.02 & 2.8 & -20.2 & 2.4 \\
\hline $9 X-5$ & 76.22 & 10.6 & -20.5 & 3.2 \\
\hline $9 X-5$ & 76.42 & 16.4 & -20.5 & 3.8 \\
\hline $9 X-5$ & 76.82 & 10.7 & -20.5 & 4.3 \\
\hline $9 X-5$ & 77.02 & 9.2 & -20.9 & 3.0 \\
\hline $9 X-5$ & 77.22 & 4.6 & -22.3 & 2.5 \\
\hline $9 X-6$ & 77.42 & 7.1 & -20.9 & 3.3 \\
\hline $9 X-6$ & 77.82 & 11.5 & -20.5 & 3.7 \\
\hline $9 X-6$ & 78.02 & 5.9 & -20.8 & 3.0 \\
\hline $9 X-6$ & 78.42 & 9.3 & -20.2 & 3.2 \\
\hline $9 X-6$ & 78.62 & 7.8 & -21.4 & 3.0 \\
\hline $9 X-7$ & 78.82 & 12.8 & -19.4 & 1.0 \\
\hline $10 \mathrm{X}-1$ & 79.42 & 10.0 & -21.0 & 7.7 \\
\hline $10 \mathrm{X}-1$ & 79.62 & 8.3 & -21.3 & 7.4 \\
\hline $10 \mathrm{X}-1$ & 79.82 & 8.8 & -21.9 & 6.3 \\
\hline $10 \mathrm{X}-1$ & 80.02 & 15.2 & -21.3 & 6.5 \\
\hline $10 \mathrm{X}-1$ & 80.22 & 9.3 & -21.5 & 5.1 \\
\hline $10 \mathrm{X}-1$ & 80.42 & 10.5 & -21.5 & 6.7 \\
\hline $10 \mathrm{X}-1$ & 80.62 & 10.7 & -20.9 & 7.4 \\
\hline $10 \mathrm{X}-1$ & 80.82 & 8.2 & -20.3 & 5.7 \\
\hline $10 X-2$ & 81.02 & 9.2 & -21.3 & 8.0 \\
\hline $10 X-2$ & 81.42 & 8.8 & -20.8 & 5.3 \\
\hline $10 X-2$ & 81.62 & 10.6 & -20.7 & 4.9 \\
\hline $10 X-2$ & 82.02 & 9.7 & -20.8 & 4.4 \\
\hline $10 X-2$ & 82.22 & 9.8 & -21.1 & 7.3 \\
\hline $10 \mathrm{X}-3$ & 82.42 & 9.0 & -20.3 & 5.6 \\
\hline $10 X-3$ & 82.62 & 13.9 & -20.4 & 5.1 \\
\hline
\end{tabular}


Table 1 (continued).

\begin{tabular}{|c|c|c|c|c|}
\hline $\begin{array}{l}\text { Core and } \\
\text { section }\end{array}$ & $\begin{array}{l}\text { Depth } \\
\text { (mbsf) }\end{array}$ & $\delta^{15} \mathrm{~N}$ & $\delta^{13} \mathrm{C}$ & $\% \mathrm{C}$ \\
\hline \multicolumn{5}{|c|}{ 117-724C-(Cont.) } \\
\hline $10 X-3$ & 82.82 & 10.6 & -21.1 & 2.7 \\
\hline $10 X-3$ & 83.02 & 11.2 & -21.0 & 3.9 \\
\hline $10 X-3$ & 83.22 & 11.2 & -20.8 & 3.6 \\
\hline $10 X-3$ & 83.42 & 7.4 & & \\
\hline $10 X-3$ & 83.82 & 12.0 & -21.2 & 2.4 \\
\hline $10 X-4$ & 84.20 & 9.8 & -20.0 & 3.8 \\
\hline $10 \times-4$ & 84.42 & 10.7 & -20.6 & 2.8 \\
\hline $10 X-4$ & 84.62 & 13.6 & -20.2 & 3.5 \\
\hline $10 X-4$ & 85.02 & 5.9 & -20.2 & 0.3 \\
\hline $10 X-4$ & 85.22 & 11.1 & -22.1 & 1.6 \\
\hline $10 X-5$ & 85.42 & 9.7 & -20.2 & 2.6 \\
\hline $10 X-5$ & 85.62 & 9.1 & -20.8 & 3.2 \\
\hline $10 X-5$ & 85.82 & 8.8 & -21.6 & 5.0 \\
\hline $10 X-5$ & 86.22 & 8.5 & -21.3 & 4.0 \\
\hline $10 X-5$ & 86.42 & 7.8 & -21.4 & 4.6 \\
\hline $10 X-5$ & 86.62 & 6.3 & -20.5 & 3.5 \\
\hline $10 \mathrm{X}-\mathrm{CC}$ & 86.77 & 5.4 & -20.7 & 1.7 \\
\hline $10 \mathrm{X}-\mathrm{CC}$ & 86.97 & 6.6 & -20.8 & 2.5 \\
\hline $11 X-1$ & 89.02 & 9.1 & -21.0 & 4.2 \\
\hline $11 X-1$ & 89.22 & 6.7 & -22.1 & 4.3 \\
\hline $11 X-1$ & 89.42 & 9.0 & -20.6 & 3.9 \\
\hline $11 X-1$ & 89.62 & 4.2 & -20.3 & 2.6 \\
\hline $11 X-1$ & 89.82 & 8.2 & -19.8 & 3.4 \\
\hline $11 X-1$ & 90.02 & 9.4 & -20.5 & 6.3 \\
\hline $11 X-2$ & 90.62 & 7.7 & -19.9 & 2.6 \\
\hline $11 X-2$ & 90.82 & 8.9 & -20.1 & 3.7 \\
\hline $11 X-2$ & 91.02 & 10.2 & -19.8 & 3.8 \\
\hline $11 X-2$ & 91.22 & 11.7 & -20.0 & 5.1 \\
\hline $11 X-2$ & 91.42 & 9.0 & -19.7 & 4.6 \\
\hline $11 X-2$ & 91.62 & 6.7 & -20.2 & 4.1 \\
\hline $11 X-2$ & 91.82 & 10.0 & -20.2 & 4.4 \\
\hline $11 X-3$ & 92.02 & 5.8 & -19.1 & 5.3 \\
\hline $11 X-3$ & 92.22 & 7.5 & -19.8 & 5.0 \\
\hline $11 X-3$ & 92.42 & 9.2 & -19.8 & 3.9 \\
\hline $11 X-3$ & 92.82 & 9.5 & -19.7 & 8.4 \\
\hline $11 X-3$ & 93.02 & 11.0 & -20.2 & 7.5 \\
\hline $11 X-3$ & 93.22 & 7.0 & -20.2 & 6.3 \\
\hline $11 X-3$ & 93.42 & 7.6 & -19.8 & 5.7 \\
\hline $11 X-4$ & 93.82 & 7.5 & -19.9 & 5.9 \\
\hline $11 X-4$ & 94.02 & 7.0 & -20.3 & 5.1 \\
\hline $11 X-4$ & 94.22 & 7.2 & -19.4 & 4.4 \\
\hline $11 X-4$ & 94.42 & 7.2 & -20.2 & 3.8 \\
\hline $11 X-4$ & 94.62 & 7.6 & -19.8 & 4.0 \\
\hline $11 X-5$ & 95.02 & 6.0 & -19.8 & 4.4 \\
\hline $11 X-5$ & 95.22 & 9.2 & -20.7 & 5.0 \\
\hline $11 X-5$ & 95.42 & 9.0 & -19.6 & 6.3 \\
\hline $11 X-5$ & 95.82 & 9.9 & -20.2 & 8.4 \\
\hline $11 X-5$ & 96.02 & 12.2 & -19.9 & 5.2 \\
\hline $11 X-5$ & 96.22 & 6.1 & -21.3 & 5.8 \\
\hline $11 X-6$ & 96.62 & 9.6 & -20.3 & 4.5 \\
\hline $11 X-6$ & 96.82 & 7.5 & -20.1 & 5.6 \\
\hline $11 X-6$ & 97.42 & 8.1 & -20.6 & 5.7 \\
\hline $11 X-6$ & 97.60 & 8.9 & -21.8 & 7.8 \\
\hline $11 X-6$ & 97.82 & 8.9 & -20.5 & 9.5 \\
\hline $11 X-7$ & 98.02 & 7.3 & -21.6 & 7.5 \\
\hline $11 X-7$ & 98.22 & 10.9 & -21.4 & 6.9 \\
\hline $12 X-1$ & 98.62 & 9.1 & -20.4 & 9.8 \\
\hline $12 X-1$ & 98.82 & 7.9 & -20.2 & 5.1 \\
\hline $12 X-1$ & 99.02 & 6.3 & -19.5 & 4.5 \\
\hline $12 X-1$ & 99.42 & 7.6 & -19.7 & 3.9 \\
\hline $12 X-1$ & 99.60 & 7.6 & -20.1 & 6.1 \\
\hline $12 X-1$ & 99.80 & 5.6 & -19.8 & 4.7 \\
\hline $12 X-1$ & 100.22 & 9.2 & -19.7 & 5.9 \\
\hline $12 X-1$ & 100.44 & 11.9 & -19.7 & 6.9 \\
\hline $12 X-2$ & 100.62 & 8.1 & -20.8 & 6.5 \\
\hline $12 X-2$ & 100.82 & 9.3 & -20.6 & 4.6 \\
\hline $12 X-2$ & 101.04 & 10.4 & -20.0 & 4.9 \\
\hline $12 X-2$ & 101.22 & 8.7 & -24.4 & 9.5 \\
\hline $12 X-2$ & 101.42 & 7.0 & -20.7 & 3.3 \\
\hline $12 X-3$ & 101.62 & 10.4 & -19.9 & 2.1 \\
\hline $12 X-3$ & 101.82 & 6.9 & -19.9 & 2.4 \\
\hline $12 X-3$ & 102.42 & 7.1 & -19.7 & 2.3 \\
\hline $12 X-3$ & 102.62 & 7.8 & -19.8 & 3.9 \\
\hline $12 X-3$ & 102.82 & 4.8 & -19.8 & 3.9 \\
\hline $12 X-3$ & 103.02 & 7.3 & -19.9 & 7.7 \\
\hline
\end{tabular}

Table 1 (continued).

\begin{tabular}{|c|c|c|c|c|}
\hline $\begin{array}{l}\text { Core and } \\
\text { section }\end{array}$ & $\begin{array}{l}\text { Depth } \\
\text { (mbsf) }\end{array}$ & $\delta^{15} \mathrm{~N}$ & ${ }^{13} \mathrm{C}$ & $\% \mathrm{C}$ \\
\hline \multicolumn{5}{|c|}{ 117-724C-(Cont.) } \\
\hline $12 \times-4$ & 103.42 & 11.2 & -19.7 & 6.0 \\
\hline $12 X-4$ & 103.62 & 8.9 & -21.1 & 3.8 \\
\hline $12 \times-4$ & 103.82 & 8.7 & -20.1 & 4.5 \\
\hline $12 X-4$ & 104.02 & 8.8 & -20.6 & 4.8 \\
\hline $12 X-4$ & 104.22 & 7.9 & -20.0 & 7.7 \\
\hline $12 X-4$ & 104.42 & 6.4 & -20.1 & 8.9 \\
\hline $12 \mathrm{X}-5$ & 104.62 & 6.8 & -20.0 & 9.6 \\
\hline $12 X-5$ & 105.02 & 10.5 & -20.1 & 5.0 \\
\hline $12 X-5$ & 105.22 & 7.1 & -20.5 & 7.2 \\
\hline $12 \mathrm{X}-5$ & 105.42 & 9.2 & -20.2 & 4.5 \\
\hline $12 X-5$ & 105.62 & 9.2 & -20.3 & 5.8 \\
\hline $12 X-5$ & 105.82 & 7.7 & -20.5 & 3.3 \\
\hline $12 \mathrm{X}-6$ & 106.23 & 4.5 & -20.3 & 3.8 \\
\hline $12 \mathrm{X}-6$ & 106.42 & 4.8 & -20.2 & 4.8 \\
\hline $12 \mathrm{X}-6$ & 106.62 & 5.9 & -20.4 & 5.2 \\
\hline $12 \mathrm{X}-6$ & 106.82 & 8.7 & -20.6 & 5.4 \\
\hline $12 \mathrm{X}-6$ & 107.02 & 8.9 & -20.7 & 6.3 \\
\hline $12 \mathrm{X}-6$ & 107.22 & 7.1 & -19.9 & 6.5 \\
\hline $12 \mathrm{X}-6$ & 107.42 & 7.6 & -20.0 & 8.2 \\
\hline $12 \mathrm{X}-7$ & 107.62 & 7.2 & -20.4 & 7.7 \\
\hline $12 X-7$ & 107.82 & 6.9 & -20.1 & 6.5 \\
\hline $13 \mathrm{X}-1$ & 108.32 & 8.7 & -20.3 & 7.9 \\
\hline $13 X-1$ & 108.52 & 6.6 & -22.2 & 8.1 \\
\hline $13 \mathrm{X}-1$ & 108.72 & 8.6 & -20.0 & 11.4 \\
\hline $13 \mathrm{X}-1$ & 108.92 & 7.3 & -22.0 & 7.3 \\
\hline $13 \mathrm{X}-1$ & 109.10 & 7.9 & -22.1 & 6.1 \\
\hline $13 \mathrm{X}-1$ & 109.52 & 8.6 & -20.9 & 7.1 \\
\hline $13 X-2$ & 109.92 & 7.6 & -21.9 & 5.7 \\
\hline $13 X-2$ & 110.12 & 10.3 & -20.3 & 8.4 \\
\hline $13 X-2$ & 110.32 & 7.6 & -19.8 & 7.2 \\
\hline $13 X-2$ & 110.52 & 9.2 & -20.4 & 9.0 \\
\hline $13 X-2$ & 110.72 & 7.9 & -21.2 & 6.6 \\
\hline $13 X-2$ & 110.94 & 7.7 & -20.5 & 8.4 \\
\hline $13 X-2$ & 111.12 & 5.5 & -20.0 & 7.1 \\
\hline $13 X-3$ & 111.32 & 5.5 & -20.3 & 6.5 \\
\hline $13 X-3$ & 111.72 & 10.0 & -20.1 & 7.5 \\
\hline $13 X-3$ & 111.92 & 9.0 & -20.3 & 5.7 \\
\hline $13 X-3$ & 112.12 & 7.5 & -20.5 & 8.8 \\
\hline $13 X-3$ & 112.32 & 8.5 & -21.9 & 6.3 \\
\hline $13 X-3$ & 112.52 & 8.9 & -22.1 & 8.5 \\
\hline $13 X-4$ & 112.92 & 8.5 & -20.3 & 11.1 \\
\hline $13 X-4$ & 113.12 & 9.1 & -20.8 & 8.4 \\
\hline $13 X-4$ & 113.32 & 7.8 & -20.5 & 8.2 \\
\hline $13 X-4$ & 113.52 & 11.0 & -20.2 & 8.9 \\
\hline $13 X-4$ & 113.72 & 10.1 & -20.6 & 12.7 \\
\hline $13 X-4$ & 113.92 & 9.7 & -20.3 & 7.0 \\
\hline $13 X-5$ & 114.32 & 8.7 & -20.1 & 6.1 \\
\hline $13 X-5$ & 114.52 & 9.6 & -19.9 & 7.5 \\
\hline $13 X-5$ & 114.72 & 7.3 & -22.5 & 4.2 \\
\hline $13 X-5$ & 115.14 & 7.3 & -20.0 & 6.6 \\
\hline $13 X-5$ & 115.32 & 5.4 & -21.9 & 6.3 \\
\hline $13 X-5$ & 115.52 & 5.4 & -20.6 & 5.5 \\
\hline $13 X-6$ & 115.92 & 6.5 & -20.1 & 6.0 \\
\hline $13 X-6$ & 116.10 & 7.7 & -20.2 & 8.0 \\
\hline $13 X-6$ & 116.32 & 5.8 & -20.5 & 7.3 \\
\hline $13 X-6$ & 116.52 & 5.7 & -20.3 & 3.4 \\
\hline $13 X-6$ & 116.72 & 6.6 & -20.7 & 5.5 \\
\hline $13 X-6$ & 116.92 & 5.8 & -22.5 & 3.9 \\
\hline $13 X-7$ & 117.32 & 7.6 & -20.5 & 7.2 \\
\hline $13 X-7$ & 117.52 & 7.6 & -20.0 & 5.1 \\
\hline $13 X-7$ & 117.72 & 7.4 & -22.2 & 4.7 \\
\hline $14 \mathrm{X}-1$ & 117.82 & 10.8 & -20.2 & 7.5 \\
\hline $14 \mathrm{X}-1$ & 118.40 & 8.6 & -20.3 & 7.1 \\
\hline $14 \mathrm{X}-1$ & 118.67 & 1.0 & -20.2 & 6.4 \\
\hline $14 \mathrm{X}-1$ & 118.81 & 9.0 & -20.9 & 6.6 \\
\hline $14 X-1$ & 119.02 & 9.6 & -20.1 & 5.3 \\
\hline $14 X-2$ & 119.42 & 10.2 & -20.1 & 7.1 \\
\hline $14 X-2$ & 119.60 & 8.4 & -20.4 & 5.7 \\
\hline $14 X-2$ & 120.02 & 8.8 & -21.3 & 4.8 \\
\hline $14 \mathrm{X}-2$ & 120.38 & 7.0 & -20.4 & 7.2 \\
\hline $14 X-3$ & 120.87 & 6.4 & -21.6 & 5.1 \\
\hline $14 \mathrm{X}-3$ & 121.63 & 7.1 & -20.3 & 6.8 \\
\hline $14 X-3$ & 121.81 & 10.2 & -20.4 & 5.7 \\
\hline $14 X-3$ & 122.02 & 6.8 & -20.5 & 6.5 \\
\hline $14 X-4$ & 122.42 & 8.5 & -20.9 & 5.7 \\
\hline
\end{tabular}


Table 1 (continued).

\begin{tabular}{|c|c|c|c|c|}
\hline $\begin{array}{l}\text { Core and } \\
\text { section }\end{array}$ & $\begin{array}{l}\text { Depth } \\
\text { (mbsf) }\end{array}$ & $\delta^{15} \mathrm{~N}$ & $\delta^{13} \mathrm{C}$ & $\% \mathrm{C}$ \\
\hline \multicolumn{5}{|c|}{ 117-724C-(Cont.) } \\
\hline $14 X-4$ & 122.62 & 7.4 & -20.7 & 5.8 \\
\hline $14 X-4$ & 122.82 & 7.9 & -23.0 & 3.8 \\
\hline $14 X-4$ & 123.02 & 6.6 & -22.4 & 4.4 \\
\hline $14 X-4$ & 123.42 & 7.5 & -22.8 & 3.3 \\
\hline $14 X-4$ & 123.62 & 9.3 & -20.0 & 5.2 \\
\hline $14 X-5$ & 123.82 & 7.5 & -20.5 & 5.2 \\
\hline $14 X-5$ & 124.23 & 6.8 & -20.4 & 3.7 \\
\hline $14 X-5$ & 124.42 & 9.7 & -20.5 & 4.1 \\
\hline $14 X-5$ & 124.62 & 8.0 & -20.2 & 5.8 \\
\hline $14 X-5$ & 125.02 & 7.6 & -22.0 & 5.9 \\
\hline $14 X-5$ & 125.22 & 7.8 & -20.0 & 4.1 \\
\hline $14 X-6$ & 125.42 & 9.5 & -20.0 & 3.0 \\
\hline $14 X-6$ & 125.62 & 4.6 & -20.4 & 2.6 \\
\hline $15 X-1$ & 127.42 & 6.3 & -21.0 & 4.5 \\
\hline $15 X-1$ & 127.62 & 7.8 & -21.9 & 3.6 \\
\hline $15 X-1$ & 128.02 & 7.4 & -22.1 & 4.7 \\
\hline $15 X-1$ & 128.20 & 6.2 & -20.9 & 5.4 \\
\hline $15 \mathrm{X}-1$ & 128.60 & 5.7 & -21.1 & 4.0 \\
\hline $15 X-2$ & 129.02 & 5.4 & -21.2 & 3.3 \\
\hline $15 X-2$ & 129.22 & 5.8 & -20.7 & 4.3 \\
\hline $15 X-2$ & 129.65 & 5.7 & -20.3 & 4.4 \\
\hline $15 X-2$ & 129.83 & 6.0 & -20.1 & 4.9 \\
\hline $15 X-3$ & 130.42 & 5.4 & -21.0 & 4.0 \\
\hline $15 X-3$ & 130.60 & 6.9 & -20.2 & 5.3 \\
\hline $15 X-3$ & 130.84 & 7.1 & -21.4 & 4.0 \\
\hline $15 X-3$ & 131.00 & 6.1 & -20.4 & 4.6 \\
\hline $15 X-3$ & 131.20 & 6.1 & -20.8 & 4.2 \\
\hline $15 X-3$ & 131.43 & 6.1 & -21.5 & 3.4 \\
\hline $15 X-3$ & 131.52 & 5.3 & -20.7 & 3.6 \\
\hline $15 X-3$ & 131.60 & 6.1 & -21.0 & 4.3 \\
\hline $15 X-4$ & 132.00 & 6.8 & -20.6 & 4.0 \\
\hline $15 X-4$ & 132.45 & 6.2 & -20.7 & 6.2 \\
\hline $15 X-4$ & 132.61 & 7.5 & -20.3 & 3.1 \\
\hline $15 X-5$ & 133.42 & 4.1 & -20.4 & 1.8 \\
\hline $15 X-5$ & 133.80 & 3.7 & -19.9 & 3.0 \\
\hline $15 X-5$ & 134.00 & 4.2 & -20.7 & 3.6 \\
\hline $15 X-5$ & 134.20 & 6.5 & -21.5 & 7.8 \\
\hline $15 X-5$ & 134.40 & 4.8 & -20.2 & 5.6 \\
\hline $15 X-5$ & 134.65 & 4.8 & -21.5 & 3.4 \\
\hline $15 X-5$ & 134.74 & 7.4 & -21.6 & 6.0 \\
\hline $15 X-5$ & 134.82 & 8.3 & -21.6 & 10.2 \\
\hline $15 X-6$ & 135.00 & 5.6 & -20.1 & 4.9 \\
\hline $15 X-6$ & 135.22 & 6.3 & -21.7 & 4.2 \\
\hline $15 X-6$ & 135.40 & 7.2 & -21.4 & 5.5 \\
\hline $15 X-6$ & 135.72 & 7.0 & -21.1 & 5.1 \\
\hline $15 X-6$ & 135.94 & 6.7 & -20.7 & 5.5 \\
\hline $15 X-7$ & 136.42 & 7.7 & -22.8 & 4.5 \\
\hline $15 X-7$ & 136.62 & 7.9 & -21.9 & 4.8 \\
\hline $15 X-7$ & 136.75 & 5.6 & -23.0 & 5.0 \\
\hline $16 X-1$ & 137.02 & 5.0 & -21.7 & 12.4 \\
\hline $16 X-1$ & 137.22 & 3.4 & -22.3 & 9.8 \\
\hline $16 X-1$ & 137.42 & 7.3 & -21.7 & 9.6 \\
\hline $16 X-1$ & 137.62 & 7.9 & -19.7 & 10.2 \\
\hline $16 X-1$ & 137.82 & 7.2 & -22.6 & 8.1 \\
\hline $16 X-1$ & 138.02 & 6.1 & -21.2 & 8.1 \\
\hline $16 X-1$ & 138.42 & 6.2 & -22.7 & 7.4 \\
\hline $16 X-2$ & 138.62 & 6.0 & -22.1 & 9.5 \\
\hline $16 X-2$ & 138.82 & 7.6 & -21.7 & 8.3 \\
\hline $16 X-2$ & 139.02 & 7.5 & -21.0 & 9.1 \\
\hline $16 X-2$ & 139.22 & 5.4 & -20.9 & 6.7 \\
\hline $16 X-2$ & 139.42 & 6.0 & -21.3 & 5.7 \\
\hline $16 X-2$ & 139.60 & 5.5 & -21.6 & 5.4 \\
\hline $16 X-2$ & 139.82 & 5.8 & -21.3 & 6.4 \\
\hline $16 X-3$ & 140.02 & 6.0 & -23.0 & 5.2 \\
\hline $16 X-3$ & 140.22 & 5.9 & -20.9 & 6.9 \\
\hline $16 \mathrm{X}-3$ & 140.62 & 4.0 & -20.7 & 7.5 \\
\hline $16 \mathrm{X}-3$ & 140.82 & 4.5 & -20.2 & 5.9 \\
\hline $16 \mathrm{X}-3$ & 141.02 & 4.9 & -20.8 & 6.3 \\
\hline $16 X-3$ & 141.22 & 6.6 & -20.0 & 6.3 \\
\hline $16 X-4$ & 141.60 & 7.1 & -21.2 & 7.0 \\
\hline $16 X-4$ & 141.82 & 7.1 & -22.8 & 4.9 \\
\hline $16 X-4$ & 142.02 & 5.5 & -22.0 & 5.1 \\
\hline $16 X-4$ & 142.22 & 4.7 & -20.6 & 9.5 \\
\hline $16 X-4$ & 142.40 & 5.9 & -20.4 & 6.8 \\
\hline $16 X-4$ & 142.60 & 5.5 & -20.6 & 7.9 \\
\hline $16 X-4$ & 142.82 & 5.4 & -20.5 & 8.7 \\
\hline
\end{tabular}

Table 1 (continued).

\begin{tabular}{|c|c|c|c|c|}
\hline $\begin{array}{l}\text { Core and } \\
\text { section }\end{array}$ & $\begin{array}{l}\text { Depth } \\
\text { (mbsf) }\end{array}$ & $\delta^{15} \mathrm{~N}$ & $\delta^{13} \mathrm{C}$ & $\% \mathrm{C}$ \\
\hline \multicolumn{5}{|c|}{ 117-724C-(Cont.) } \\
\hline $16 X-5$ & 143.07 & 6.6 & -20.7 & 5.5 \\
\hline $16 X-5$ & 143.23 & 5.5 & -21.3 & 5.2 \\
\hline $16 X-5$ & 143.40 & 7.3 & -21.1 & 5.4 \\
\hline $16 X-5$ & 143.62 & 5.3 & -20.4 & 7.9 \\
\hline $16 X-5$ & 144.02 & 6.7 & -20.4 & 7.9 \\
\hline $16 X-5$ & 144.22 & 5.2 & -20.6 & 7.4 \\
\hline $16 X-5$ & 144.42 & 5.8 & -20.3 & 8.0 \\
\hline $16 X-6$ & 144.62 & 4.1 & -20.8 & 4.1 \\
\hline $16 X-6$ & 144.82 & 5.8 & -22.7 & 4.9 \\
\hline $16 X-6$ & 144.98 & 3.5 & -20.3 & 6.4 \\
\hline $16 X-6$ & 145.22 & 5.2 & -20.9 & 6.9 \\
\hline $16 X-6$ & 145.42 & 5.1 & -20.7 & 6.9 \\
\hline $16 X-6$ & 145.62 & 5.3 & -20.6 & 6.7 \\
\hline $16 X-6$ & 145.82 & 5.6 & -21.0 & 6.4 \\
\hline $16 X-7$ & 146.02 & 3.9 & -20.9 & 3.9 \\
\hline $16 \times-7$ & 146.22 & 6.5 & -21.0 & 6.5 \\
\hline $16 \times-7$ & 146.42 & 4.5 & -21.0 & 5.9 \\
\hline $17 \mathrm{X}-1$ & 146.62 & 8.2 & -19.8 & 7.0 \\
\hline $17 X-1$ & 146.82 & 6.2 & -19.7 & 6.4 \\
\hline $17 X-1$ & 147.02 & 7.9 & -20.5 & 7.1 \\
\hline $17 X-1$ & 147.22 & 7.9 & -19.7 & 7.2 \\
\hline $17 X-1$ & 148.02 & 6.5 & -19.9 & 7.5 \\
\hline $17 X-2$ & 148.20 & 6.3 & -19.7 & 7.5 \\
\hline $17 X-2$ & 148.42 & 8.2 & -19.7 & 7.9 \\
\hline $17 X-2$ & 148.62 & 10.3 & -20.1 & 9.3 \\
\hline $17 X-2$ & 148.81 & 7.9 & -22.5 & 4.3 \\
\hline $17 X-3$ & 149.62 & 5.6 & -21.0 & 8.6 \\
\hline $17 X-3$ & 149.82 & 8.5 & -20.1 & 9.1 \\
\hline $17 X-3$ & 150.02 & 6.7 & -22.4 & 8.2 \\
\hline $17 X-3$ & 150.22 & 6.8 & -20.0 & 11.9 \\
\hline $17 X-3$ & 150.42 & 8.1 & -20.0 & 10.8 \\
\hline $17 X-3$ & 150.62 & 6.2 & -20.2 & 10.3 \\
\hline $17 X-3$ & 150.82 & 4.4 & -21.2 & 9.9 \\
\hline $17 X-3$ & 151.02 & 6.6 & -19.7 & 8.2 \\
\hline $17 X-4$ & 151.20 & 6.6 & -21.7 & 6.4 \\
\hline $17 X-4$ & 151.42 & 6.8 & -19.9 & 8.2 \\
\hline $17 X-4$ & 151.48 & 7.4 & -19.9 & 8.8 \\
\hline $17 X-4$ & 152.22 & 9.1 & -19.9 & 6.0 \\
\hline $17 X-4$ & 152.42 & 7.2 & -21.3 & 6.9 \\
\hline $17 X-5$ & 152.62 & 6.9 & -19.9 & 9.3 \\
\hline $17 X-5$ & 152.82 & 4.4 & -20.2 & 10.9 \\
\hline $17 X-5$ & 153.02 & 8.3 & -20.2 & 6.3 \\
\hline $17 X-5$ & 153.22 & 7.1 & -21.8 & 5.0 \\
\hline $17 X-5$ & 153.42 & 9.1 & -20.2 & 5.6 \\
\hline $17 X-5$ & 153.62 & 6.1 & -21.0 & 5.9 \\
\hline $17 X-5$ & 153.82 & 8.9 & -20.2 & 5.6 \\
\hline $17 X-6$ & 154.22 & 9.9 & -20.2 & 6.1 \\
\hline $17 X-6$ & 154.42 & 14.0 & -20.0 & 6.0 \\
\hline $17 X-6$ & 154.62 & 10.5 & -20.6 & 3.3 \\
\hline $17 X-6$ & 154.82 & 7.5 & -20.4 & 7.3 \\
\hline $17 X-6$ & 155.02 & 8.3 & -20.2 & 4.1 \\
\hline $18 \mathrm{X}-1$ & 156.32 & 7.5 & -20.2 & 6.8 \\
\hline $18 \mathrm{X}-1$ & 156.52 & 7.3 & -20.8 & 7.7 \\
\hline $18 \mathrm{X}-1$ & 156.72 & 7.3 & -20.4 & 7.3 \\
\hline $18 \mathrm{X}-1$ & 156.92 & 6.8 & -20.7 & 6.3 \\
\hline $18 X-1$ & 157.12 & 5.9 & -20.3 & 6.6 \\
\hline $18 \mathrm{X}-1$ & 157.32 & 1.5 & -20.3 & 6.1 \\
\hline $18 \mathrm{X}-1$ & 157.52 & 7.6 & -20.8 & 6.2 \\
\hline $18 \mathrm{X}-1$ & 157.68 & 7.5 & -20.6 & 7.8 \\
\hline $18 X-2$ & 157.92 & 6.5 & -20.8 & 9.4 \\
\hline $18 \mathrm{X}-2$ & 158.10 & 7.2 & -20.6 & 6.5 \\
\hline $18 X-2$ & 158.72 & 7.0 & -20.1 & 5.6 \\
\hline $18 X-2$ & 158.92 & 6.5 & -20.1 & 6.1 \\
\hline $18 X-2$ & 159.12 & 6.0 & -20.9 & 6.4 \\
\hline $18 X-3$ & 159.32 & 4.9 & -21.0 & 7.5 \\
\hline $18 X-3$ & 159.52 & 7.0 & -20.5 & 5.9 \\
\hline $18 X-3$ & 159.72 & 8.0 & -20.9 & 10.6 \\
\hline $18 X-3$ & 159.92 & 9.2 & -22.1 & 9.2 \\
\hline $18 X-3$ & 160.12 & 7.4 & -20.3 & 10.0 \\
\hline $18 X-3$ & 160.32 & 6.1 & -20.9 & 7.3 \\
\hline $18 X-3$ & 160.52 & 6.9 & -19.9 & 4.7 \\
\hline $18 X-4$ & 160.95 & 7.5 & -20.1 & 5.0 \\
\hline $18 X-4$ & 161.12 & 5.7 & -21.5 & 6.7 \\
\hline $18 X-4$ & 161.32 & 7.5 & -21.0 & 6.6 \\
\hline $18 X-4$ & 161.72 & 9.5 & -19.9 & 4.0 \\
\hline $18 X-4$ & 161.92 & 5.7 & -20.5 & 6.2 \\
\hline
\end{tabular}


Table 1 (continued).

\begin{tabular}{|c|c|c|c|c|}
\hline $\begin{array}{l}\text { Core and } \\
\text { section }\end{array}$ & $\begin{array}{l}\text { Depth } \\
\text { (mbsf) }\end{array}$ & $\delta^{15} \mathrm{~N}$ & $\delta^{13} \mathrm{C}$ & $\% \mathrm{C}$ \\
\hline \multicolumn{5}{|c|}{ 117-724C-(Cont.) } \\
\hline $18 X-4$ & 162.12 & 5.7 & -19.9 & 11.4 \\
\hline $18 X-5$ & 162.32 & 7.2 & -20.0 & 10.6 \\
\hline $18 X-5$ & 162.52 & 6.1 & -20.5 & 6.4 \\
\hline $18 X-5$ & 163.12 & 6.5 & -21.4 & 6.1 \\
\hline $18 X-5$ & 163.32 & 5.7 & -19.9 & 2.1 \\
\hline $18 X-5$ & 163.72 & 6.5 & -20.4 & 6.2 \\
\hline $18 \mathrm{X}-6$ & 163.92 & 8.3 & -20.2 & 4.0 \\
\hline $18 \mathrm{X}-6$ & 164.12 & 5.8 & -20.0 & 4.0 \\
\hline $18 \mathrm{X}-6$ & 164.52 & 5.9 & -20.2 & 3.6 \\
\hline $18 X-6$ & 164.72 & 5.3 & -20.1 & 6.3 \\
\hline $18 X-6$ & 164.92 & 6.2 & -19.7 & 4.3 \\
\hline $18 \mathrm{X}-6$ & 165.12 & 5.2 & -20.0 & 4.0 \\
\hline $19 \mathrm{X}-1$ & 166.09 & 6.8 & -20.2 & 9.1 \\
\hline $19 \mathrm{X}-1$ & 166.31 & 6.7 & -20.2 & 8.9 \\
\hline $19 \mathrm{X}-1$ & 166.50 & 5.5 & -20.3 & 6.4 \\
\hline $19 \mathrm{X}-1$ & 167.14 & 7.2 & -20.2 & 5.9 \\
\hline $19 \mathrm{X}-1$ & 167.32 & 6.7 & -19.9 & 8.6 \\
\hline $19 X-2$ & 167.52 & 6.3 & -21.2 & 6.7 \\
\hline $19 \mathrm{X}-2$ & 167.72 & 5.7 & -19.9 & 7.7 \\
\hline $19 X-2$ & 167.92 & 5.2 & -20.6 & 5.9 \\
\hline $19 \mathrm{X}-2$ & 168.12 & 5.8 & -20.3 & 4.1 \\
\hline $19 X-2$ & 168.25 & 7.3 & -20.5 & 7.9 \\
\hline $19 \mathrm{X}-2$ & 168.52 & 6.7 & -21.2 & 1.0 \\
\hline $19 \mathrm{X}-2$ & 168.72 & 7.4 & -19.6 & 8.2 \\
\hline $20 \mathrm{X}-1$ & 175.54 & 8.4 & -19.7 & 5.4 \\
\hline $20 \mathrm{X}-1$ & 175.72 & 5.5 & -19.7 & 8.8 \\
\hline $20 \mathrm{X}-1$ & 175.92 & 8.2 & -19.3 & 6.6 \\
\hline $20 X-1$ & 176.36 & 8.9 & -19.7 & 5.5 \\
\hline $20 \mathrm{X}-1$ & 176.52 & 5.2 & -20.3 & 6.9 \\
\hline $20 \mathrm{X}-1$ & 176.74 & 8.3 & -19.7 & 6.1 \\
\hline $20 \mathrm{X}-1$ & 176.92 & 6.1 & -20.0 & 5.1 \\
\hline $20 \mathrm{X}-2$ & 177.12 & 8.6 & -20.0 & 11.0 \\
\hline $20 \mathrm{X}-2$ & 177.32 & 8.8 & -19.7 & 8.1 \\
\hline $20 \mathrm{X}-2$ & 177.72 & 5.4 & -19.7 & 7.3 \\
\hline $20 \mathrm{X}-2$ & 178.12 & 9.4 & -20.0 & 5.7 \\
\hline $20 \mathrm{X}-2$ & 178.36 & 6.5 & -21.2 & 4.5 \\
\hline $20 \mathrm{X}-3$ & 178.52 & 4.7 & -20.3 & 3.8 \\
\hline $20 \mathrm{X}-3$ & 178.72 & 7.5 & -19.9 & 3.9 \\
\hline $20 \mathrm{X}-3$ & 178.92 & 7.5 & -19.8 & 4.4 \\
\hline $20 \mathrm{X}-3$ & 179.29 & 7.7 & -20.7 & 6.2 \\
\hline $20 \mathrm{X}-3$ & 179.52 & 6.3 & -19.8 & 7.9 \\
\hline $20 \mathrm{X}-3$ & 179.72 & 5.6 & -21.7 & 6.7 \\
\hline $20 \mathrm{X}-3$ & 179.92 & 6.9 & -20.2 & 11.6 \\
\hline $20 \times-4$ & 180.12 & 11.3 & -20.4 & 10.7 \\
\hline $20 \times-4$ & 180.32 & 7.4 & -20.8 & 5.7 \\
\hline $20 \times-4$ & 180.52 & 8.7 & -20.4 & 7.6 \\
\hline $20 \times-4$ & 180.72 & 6.2 & -20.5 & 2.8 \\
\hline $20 \times-4$ & 181.12 & 3.7 & -20.5 & 3.0 \\
\hline $20 \times-4$ & 181.32 & 5.6 & -19.5 & 3.3 \\
\hline $20 X-5$ & 181.52 & 3.6 & -19.4 & 4.4 \\
\hline $20 \mathrm{X}-5$ & 181.72 & 9.4 & -19.4 & 4.5 \\
\hline $20 X-5$ & 181.92 & 7.6 & & 5.0 \\
\hline $20 X-5$ & 182.12 & 5.3 & -19.5 & 5.5 \\
\hline $20 \mathrm{X}-5$ & 182.32 & 6.4 & -19.3 & 5.8 \\
\hline $20 X-5$ & 182.52 & 6.4 & -19.6 & 7.7 \\
\hline $20 X-5$ & 182.72 & 4.4 & -19.3 & 5.5 \\
\hline $20 X-5$ & 182.92 & 7.4 & -19.5 & 5.9 \\
\hline $20 \mathrm{X}-6$ & 183.12 & 6.1 & -19.9 & 9.9 \\
\hline $20 \mathrm{X}-6$ & 183.32 & 4.9 & -20.4 & 11.1 \\
\hline $20 \mathrm{X}-6$ & 183.52 & 7.7 & -20.6 & 11.4 \\
\hline $20 X-6$ & 183.72 & 5.5 & -20.5 & 18.2 \\
\hline $20 \mathrm{X}-6$ & 183.92 & 5.6 & -22.5 & 8.2 \\
\hline $21 X-1$ & 185.22 & 7.2 & -20.9 & 10.9 \\
\hline $21 X-1$ & 185.62 & 5.4 & -20.5 & 10.5 \\
\hline $21 X-1$ & 185.85 & 5.8 & -22.0 & 6.3 \\
\hline$X-1$ & 186.02 & 7.8 & -19.8 & 11.1 \\
\hline$X-1$ & 186.22 & 10.7 & -20.4 & 8.7 \\
\hline $21 \mathrm{X}-1$ & 186.42 & 9.3 & -20.5 & 7.5 \\
\hline $21 X-1$ & 186.62 & 10.5 & -20.5 & 8.6 \\
\hline $21 X-2$ & 186.82 & 13.3 & -21.4 & 17.2 \\
\hline $21 X-2$ & 187.02 & 6.2 & -20.0 & 7.2 \\
\hline $21 X-2$ & 187.22 & 5.0 & -21.6 & 5.5 \\
\hline & 187.42 & 7.3 & -21.2 & 6.2 \\
\hline $21 \mathrm{X}-2$ & 188.02 & 7.6 & -21.4 & 8.9 \\
\hline $21 X-3$ & 188.22 & 8.3 & -19.9 & 14.5 \\
\hline $21 X-3$ & 189.02 & 9.4 & -19.8 & 14.0 \\
\hline
\end{tabular}

Table 1 (continued).

\begin{tabular}{|c|c|c|c|c|}
\hline $\begin{array}{l}\text { Core and } \\
\text { section }\end{array}$ & $\begin{array}{l}\text { Depth } \\
\text { (mbsf) }\end{array}$ & $\delta^{15} \mathrm{~N}$ & $\delta^{13} \mathrm{C}$ & $\% \mathrm{C}$ \\
\hline \multicolumn{5}{|c|}{$117-724 \mathrm{C}-($ Cont.) } \\
\hline $21 X-3$ & 189.22 & 8.0 & -20.5 & 9.6 \\
\hline $21 \times-4$ & 189.82 & 6.6 & -22.5 & 4.9 \\
\hline $21 \times-4$ & 190.02 & 5.9 & -20.8 & 9.2 \\
\hline $21 X-4$ & 190.22 & 7.1 & -20.0 & 9.8 \\
\hline $21 X-4$ & 190.42 & 7.2 & -20.2 & 11.9 \\
\hline $22 \mathrm{X}-1$ & 194.82 & 3.9 & $-20: 3$ & 9.7 \\
\hline $22 \mathrm{X}-1$ & 195.02 & 7.6 & -21.0 & 12.1 \\
\hline $22 \mathrm{X}-1$ & 195.42 & 6.5 & -20.5 & 13.0 \\
\hline $22 \mathrm{X}-1$ & 195.62 & 6.8 & -19.7 & 14.0 \\
\hline $22 X-1$ & 195.82 & 6.8 & -19.7 & 9.6 \\
\hline $22 \mathrm{X}-1$ & 196.02 & 8.4 & -20.2 & 9.6 \\
\hline $22 \mathrm{X}-1$ & 196.22 & 6.4 & -19.8 & 6.7 \\
\hline $22 \mathrm{X}-2$ & 196.40 & 7.1 & -19.9 & 5.1 \\
\hline $22 \mathrm{X}-2$ & 196.62 & 6.2 & -20.7 & 8.1 \\
\hline $22 \mathrm{X}-2$ & 196.82 & 6.1 & -20.1 & 10.2 \\
\hline $22 \mathrm{X}-2$ & 197.02 & 5.9 & -20.6 & 12.6 \\
\hline $22 \mathrm{X}-2$ & 197.22 & 7.0 & -19.8 & 10.9 \\
\hline $22 X-2$ & 197.42 & 7.5 & -20.4 & 11.5 \\
\hline $22 \mathrm{X}-2$ & 197.62 & 4.8 & -22.1 & 9.9 \\
\hline $22 \mathrm{X}-3$ & 197.82 & 7.5 & -21.0 & 9.7 \\
\hline $22 \mathrm{X}-3$ & 198.02 & 3.9 & -21.0 & 8.9 \\
\hline $22 \mathrm{X}-3$ & 198.22 & 8.9 & -19.3 & 13.3 \\
\hline $22 \mathrm{X}-3$ & 198.42 & 5.6 & -19.2 & 4.6 \\
\hline $22 \mathrm{X}-3$ & 198.62 & & -19.9 & 12.8 \\
\hline $22 \mathrm{X}-3$ & 198.82 & 7.8 & -20.9 & 10.8 \\
\hline $22 \mathrm{X}-3$ & 199.02 & 6.5 & -20.6 & 12.6 \\
\hline $22 \mathrm{X}-3$ & 199.22 & 7.6 & -21.1 & 10.9 \\
\hline $22 X-4$ & 199.42 & 6.9 & -18.9 & 9.8 \\
\hline $22 X-4$ & 199.65 & 7.4 & -19.9 & 14.1 \\
\hline $22 X-4$ & 199.86 & 7.8 & -19.9 & 11.3 \\
\hline $22 X-4$ & 200.42 & 7.6 & -20.5 & 11.1 \\
\hline $22 X-4$ & 200.62 & 7.0 & -18.6 & 12.8 \\
\hline $22 X-5$ & 200.82 & 6.2 & -20.1 & 11.5 \\
\hline $22 X-5$ & 201.82 & 7.4 & -19.9 & 10.5 \\
\hline $22 X-5$ & 201.83 & 7.2 & -20.4 & 12.4 \\
\hline $22 \mathrm{X}-5$ & 202.28 & 6.4 & -20.8 & 13.0 \\
\hline $22 \mathrm{X}-6$ & 203.22 & 6.9 & -21.1 & 15.9 \\
\hline $22 \mathrm{X}-6$ & 203.62 & 6.7 & -20.3 & 7.8 \\
\hline $22 X-7$ & 203.82 & 6.4 & -20.2 & 8.7 \\
\hline $22 X-7$ & 203.97 & 6.9 & -20.3 & 9.8 \\
\hline
\end{tabular}

$117-725 \mathrm{C}$ -

$\begin{array}{lrrrr}1 \mathrm{H}-1 & 0.20 & 6.9 & -20.7 & 1.3 \\ 1 \mathrm{H}-1 & 0.40 & 9.8 & -19.4 & 1.0 \\ 1 \mathrm{H}-1 & 0.60 & 8.2 & -22.8 & 1.0 \\ 1 \mathrm{H}-1 & 0.80 & 11.8 & -19.5 & 0.7 \\ 1 \mathrm{H}-1 & 1.20 & 10.9 & -19.5 & 0.5 \\ 1 \mathrm{H}-2 & 1.40 & 11.3 & -19.8 & 0.6 \\ 1 \mathrm{H}-2 & 1.60 & 7.7 & -20.1 & 1.0 \\ 1 \mathrm{H}-2 & 1.80 & 12.2 & -19.4 & 0.6 \\ 1 \mathrm{H}-2 & 2.00 & 14.0 & -19.2 & 0.5 \\ 1 \mathrm{H}-2 & 2.20 & 15.7 & -19.1 & 0.8 \\ 1 \mathrm{H}-2 & 2.37 & 11.3 & -18.9 & 0.8 \\ 1 \mathrm{H}-2 & 2.60 & 9.9 & -18.8 & 0.9 \\ 1 \mathrm{H}-3 & 3.00 & 11.6 & -18.8 & 1.4 \\ 1 \mathrm{H}-3 & 3.20 & 5.9 & -18.7 & 1.0 \\ 1 \mathrm{H}-3 & 3.40 & 13.7 & -19.0 & 0.9 \\ 1 \mathrm{H}-3 & 3.60 & 8.9 & -18.6 & 0.7 \\ 1 \mathrm{H}-3 & 3.80 & 7.2 & -20.7 & 0.9 \\ 1 \mathrm{H}-3 & 4.00 & 10.7 & -18.7 & 1.2 \\ 1 \mathrm{H}-3 & 4.20 & 15.1 & -18.5 & 1.3 \\ 1 \mathrm{H}-4 & 4.60 & 16.0 & -19.0 & 1.2 \\ 1 \mathrm{H}-4 & 4.80 & 12.3 & -19.1 & 1.2 \\ 1 \mathrm{H}-4 & 5.20 & 8.5 & -19.0 & 0.9 \\ 1 \mathrm{H}-4 & 5.40 & 6.8 & -19.1 & 1.3 \\ 1 \mathrm{H}-4 & 5.60 & 5.5 & -19.0 & 1.5 \\ 1 \mathrm{H}-4 & 5.80 & 14.5 & -19.3 & 1.0 \\ 1 \mathrm{H}-5 & 6.20 & 5.5 & -19.5 & 0.9 \\ 1 \mathrm{H}-5 & 6.40 & 9.6 & -18.7 & 0.8 \\ 1 \mathrm{H}-5 & 7.00 & 16.6 & -20.1 & 1.3 \\ 1 \mathrm{H}-5 & 7.20 & 14.8 & -19.5 & 0.9 \\ 1 \mathrm{H}-5 & 7.40 & 13.1 & -19.2 & 0.6 \\ 1 \mathrm{H}-6 & 7.80 & 6.4 & -19.4 & 0.8 \\ 1 \mathrm{H}-6 & 8.00 & 4.6 & -20.7 & 0.6 \\ 2 \mathrm{H}-1 & 9.00 & 11.3 & -20.3 & 0.5\end{array}$


Table 1 (continued).

\begin{tabular}{|c|c|c|c|c|}
\hline $\begin{array}{l}\text { Core and } \\
\text { section }\end{array}$ & $\begin{array}{l}\text { Depth } \\
\text { (mbsf) }\end{array}$ & $\delta^{15} \mathrm{~N}$ & $\delta^{13} \mathrm{C}$ & $\% \mathrm{C}$ \\
\hline \multicolumn{5}{|c|}{ 117-725C-(Cont.) } \\
\hline $2 \mathrm{H}-1$ & 9.60 & 11.3 & -19.6 & 1.0 \\
\hline $2 \mathrm{H}-1$ & 9.80 & 12.1 & -19.8 & 1.1 \\
\hline $2 \mathrm{H}-1$ & 10.00 & 11.1 & -19.9 & 1.2 \\
\hline $2 \mathrm{H}-1$ & 10.20 & 7.8 & -20.3 & 1.1 \\
\hline $2 \mathrm{H}-2$ & 10.60 & 12.7 & -19.9 & 2.0 \\
\hline $2 \mathrm{H}-2$ & 10.80 & 8.8 & -19.9 & 1.8 \\
\hline $2 \mathrm{H}-2$ & 11.20 & 9.4 & -19.6 & 0.8 \\
\hline $2 \mathrm{H}-2$ & 11.40 & 5.6 & -20.8 & 1.2 \\
\hline $2 \mathrm{H}-2$ & 11.60 & 15.2 & -19.9 & 0.9 \\
\hline $2 \mathrm{H}-2$ & 11.80 & 11.0 & -20.0 & 0.8 \\
\hline $2 \mathrm{H}-3$ & 12.00 & 14.5 & -20.3 & 0.6 \\
\hline $2 \mathrm{H}-3$ & 12.20 & 14.4 & -19.0 & 0.8 \\
\hline $2 \mathrm{H}-3$ & 12.40 & 9.4 & -20.4 & 0.9 \\
\hline $2 \mathrm{H}-3$ & 12.60 & 4.9 & -20.1 & 0.8 \\
\hline $2 \mathrm{H}-3$ & 12.80 & 13.5 & -19.4 & 1.2 \\
\hline $2 \mathrm{H}-3$ & 13.00 & 15.2 & -19.8 & 1.6 \\
\hline $2 \mathrm{H}-3$ & 13.20 & 12.0 & -18.9 & 1.6 \\
\hline $2 \mathrm{H}-3$ & 13.40 & 11.1 & -19.4 & 1.4 \\
\hline $2 \mathrm{H}-4$ & 13.60 & 8.4 & -19.6 & 1.3 \\
\hline $2 \mathrm{H}-4$ & 13.80 & 7.2 & -19.5 & 1.2 \\
\hline $2 \mathrm{H}-4$ & 14.00 & 13.3 & -19.7 & 1.4 \\
\hline $2 \mathrm{H}-4$ & 14.20 & 13.3 & -20.1 & 1.7 \\
\hline $2 \mathrm{H}-4$ & 14.60 & 7.5 & -19.8 & 0.5 \\
\hline $2 \mathrm{H}-4$ & 14.80 & 14.4 & -20.0 & 1.0 \\
\hline $2 \mathrm{H}-5$ & 15.00 & 10.0 & -19.7 & 1.1 \\
\hline $2 \mathrm{H}-5$ & 15.20 & 11.1 & -19.6 & 1.5 \\
\hline $2 \mathrm{H}-5$ & 15.40 & 13.8 & -18.1 & 1.0 \\
\hline $2 \mathrm{H}-5$ & 15.60 & 14.0 & -20.0 & 2.0 \\
\hline $2 \mathrm{H}-5$ & 16.00 & 9.9 & -19.3 & 1.1 \\
\hline $2 \mathrm{H}-5$ & 16.40 & 11.7 & -19.0 & 1.5 \\
\hline $2 \mathrm{H}-6$ & 16.60 & 7.3 & -18.6 & 0.9 \\
\hline $2 \mathrm{H}-6$ & 16.80 & 8.8 & -20.0 & 0.5 \\
\hline $2 \mathrm{H}-6$ & 17.00 & 14.1 & -19.3 & 0.5 \\
\hline $2 \mathrm{H}-6$ & 17.20 & 11.8 & -19.8 & 0.7 \\
\hline $2 \mathrm{H}-6$ & 17.40 & 5.7 & -20.4 & 0.7 \\
\hline $2 \mathrm{H}-6$ & 17.60 & 5.3 & -17.6 & 0.6 \\
\hline $2 \mathrm{H}-6$ & 17.80 & 6.5 & -19.6 & 0.8 \\
\hline $2 \mathrm{H}-7$ & 18.00 & 6.2 & -20.5 & 0.7 \\
\hline $2 \mathrm{H}-7$ & 18.20 & 13.1 & -20.2 & 0.6 \\
\hline $2 \mathrm{H}-7$ & 18.40 & 14.2 & -19.9 & 0.7 \\
\hline $3 \mathrm{H}-1$ & 18.60 & 17.0 & -20.0 & 0.6 \\
\hline $3 \mathrm{H}-1$ & 18.80 & 15.1 & -20.1 & 0.6 \\
\hline $3 \mathrm{H}-1$ & 19.00 & 13.1 & -20.0 & 0.7 \\
\hline $3 \mathrm{H}-1$ & 19.40 & 13.3 & -19.8 & 1.0 \\
\hline $3 \mathrm{H}-1$ & 20.00 & 11.2 & -19.1 & 0.7 \\
\hline $3 \mathrm{H}-2$ & 20.20 & 10.0 & -19.9 & 1.3 \\
\hline $3 \mathrm{H}-2$ & 21.00 & 16.7 & -20.1 & 0.7 \\
\hline $3 \mathrm{H}-2$ & 21.20 & 13.6 & -20.1 & 0.6 \\
\hline $3 \mathrm{H}-2$ & 21.40 & 15.0 & -20.2 & 0.6 \\
\hline $3 \mathrm{H}-3$ & 21.60 & 12.0 & -22.5 & 0.5 \\
\hline $3 \mathrm{H}-3$ & 21.80 & 13.3 & -20.1 & 0.8 \\
\hline $3 \mathrm{H}-3$ & 22.00 & 12.6 & -20.4 & 0.7 \\
\hline $3 \mathrm{H}-3$ & 22.60 & 13.7 & -20.1 & 0.6 \\
\hline $3 \mathrm{H}-3$ & 22.80 & 11.2 & -20.6 & 0.7 \\
\hline $3 \mathrm{H}-4$ & 23.20 & 13.6 & -19.9 & 0.8 \\
\hline $3 \mathrm{H}-4$ & 23.60 & 12.1 & -18.8 & 0.7 \\
\hline $3 \mathrm{H}-4$ & 23.80 & 11.7 & -19.9 & 1.2 \\
\hline $3 \mathrm{H}-4$ & 24.00 & 12.7 & -19.6 & 0.6 \\
\hline $3 \mathrm{H}-4$ & 24.20 & 11.6 & -19.9 & 0.9 \\
\hline $3 \mathrm{H}-5$ & 24.60 & 10.6 & -19.7 & 0.7 \\
\hline $3 \mathrm{H}-5$ & 24.80 & 11.1 & -20.1 & 1.2 \\
\hline $3 \mathrm{H}-5$ & 25.00 & 14.9 & -20.0 & 1.0 \\
\hline $3 \mathrm{H}-5$ & 25.20 & 10.8 & -19.3 & 1.3 \\
\hline $3 \mathrm{H}-5$ & 25.40 & 12.9 & -20.1 & 0.9 \\
\hline $3 \mathrm{H}-5$ & 25.60 & 13.5 & -20.6 & 0.5 \\
\hline $3 \mathrm{H}-5$ & 25.80 & 14.3 & -21.1 & 1.3 \\
\hline $3 \mathrm{H}-5$ & 26.00 & 10.1 & -20.1 & 0.9 \\
\hline $3 \mathrm{H}-6$ & 26.20 & 14.3 & -20.8 & 0.7 \\
\hline $3 \mathrm{H}-6$ & 26.60 & 13.9 & -20.8 & 1.4 \\
\hline $3 \mathrm{H}-6$ & 26.80 & 10.5 & -20.0 & 0.9 \\
\hline $3 \mathrm{H}-6$ & 27.00 & 14.9 & -20.2 & 0.7 \\
\hline $3 \mathrm{H}-6$ & 27.40 & 13.1 & -20.3 & 0.9 \\
\hline $3 \mathrm{H}-7$ & 27.60 & 15.4 & -21.9 & 0.5 \\
\hline $3 \mathrm{H}-7$ & 27.80 & 12.3 & -20.1 & 0.8 \\
\hline $3 \mathrm{H}-7$ & 28.00 & 14.1 & -21.8 & 0.8 \\
\hline
\end{tabular}

Table 1 (continued).

\begin{tabular}{|c|c|c|c|c|}
\hline $\begin{array}{l}\text { Core and } \\
\text { section }\end{array}$ & $\begin{array}{l}\text { Depth } \\
\text { (mbsf) }\end{array}$ & $\delta^{15} \mathrm{~N}$ & $\delta^{13} \mathrm{C}$ & $\% \mathrm{C}$ \\
\hline \multicolumn{5}{|c|}{ 117-725C-(Cont.) } \\
\hline $3 \mathrm{H}-7$ & 28.20 & 15.0 & -21.2 & 1.1 \\
\hline $4 \mathrm{X}-1$ & 28.70 & 10.0 & -25.0 & 0.2 \\
\hline $4 \mathrm{X}-1$ & 28.90 & 14.7 & -23.7 & 0.4 \\
\hline $4 X-1$ & 29.30 & 10.4 & -23.4 & 0.4 \\
\hline $4 \mathrm{X}-1$ & 29.50 & 12.2 & -24.2 & 0.4 \\
\hline $4 \mathrm{X}-1$ & 29.70 & 10.9 & -25.6 & 0.2 \\
\hline $4 X-2$ & 30.10 & 5.3 & -21.9 & 0.7 \\
\hline $4 \mathrm{X}-2$ & 30.50 & 10.3 & -21.6 & 1.5 \\
\hline $4 X-2$ & 30.70 & 10.0 & -22.7 & 0.5 \\
\hline $4 X-2$ & 30.90 & 12.8 & -20.5 & 2.1 \\
\hline $4 X-2$ & 31.10 & 12.0 & -21.6 & 1.1 \\
\hline $4 X-2$ & 31.30 & 8.2 & -19.8 & 1.0 \\
\hline $4 X-3$ & 31.50 & 14.5 & -20.6 & 1.3 \\
\hline $4 X-3$ & 31.70 & 8.3 & -20.4 & 1.0 \\
\hline $4 X-3$ & 31.90 & 10.3 & -21.6 & 0.5 \\
\hline $4 X-3$ & 32.10 & 11.3 & -20.4 & 0.3 \\
\hline
\end{tabular}

enized sample interval of $20 \mathrm{~mm}$ would include $140-570 \mathrm{yr}$ of sediment influx to the ocean floor. Organic carbon content of the acidified residue of the sediments is seen to increase drastically with depth. Highest values occur in the Pliocene, reaching a maximum of $18.2 \%$ at a depth of approximately $180 \mathrm{~m}$. A sharp decline in the organic content occurs toward the PliocenePleistocene boundary (near $130 \mathrm{~m}$ ) with an increase above that boundary. From $80 \mathrm{~m}$ to the surface of the core, a nearly constant amount of $2 \%-3 \%$ of the residue could be identified as organic carbon. The lowest amounts of carbon are observed during this period, at $0.2 \%$. The levels of carbon are noted to be much lower during the Pleistocene-Holocene section of the core, but are still much above the world average for typical carbon content of marine sediments. The average carbon content measured on these samples was $4.9 \%$ with a standard deviation of $2.9 \%$, (Fig. 2).

Stable carbon isotopic compositions of this material ranged from $-17.7 \%$ to $-24.7 \%$, with an average of $-20.4 \%$ (standard deviation $=0.8 \%$ ). The values are fairly constant but have a slight enrichment in the Pleistocene-Holocene section $(-20 \%)$ over the Pliocene $(-21 \%)$. Certain intervals of approximately $20 \mathrm{~m}$ show depletions of about $1 \%$ from the average above and below it.

Stable nitrogen compositions of these sediments are highly variable, with an average value of $8.9 \%$, range of $1.0 \% 0-18.9 \%$, and standard deviation of $2.5 \%$ (Fig. 3). The Pliocene has the most uniform values, near the average, with more enriched samples noted in the Pleistocene-Holocene. The very surface of the core had samples which were depleted to near the average. No statistically significant correlations (Pearson R) were observed between the isotopic compositions nor with the isotopic compositions and the organic carbon content (Fig. 4).

\section{Site 725}

Hole $725 \mathrm{C}$ was sampled at $200 \mathrm{~mm}$ intervals yielding a resolution of approximately $1400 \mathrm{yr}$ between samples at the estimated sedimentation rate of $143 \mathrm{~m} / \mathrm{m}$.y. Each $20 \mathrm{~mm}$ sample interval which was homogenized therefore represents $140 \mathrm{yr}$ of sedimentation. Organic carbon analysis of these samples yielded amounts approximately $1 \%$ less than levels found in the near surface portion of Hole $724 \mathrm{C}$, averaging $0.9 \% \pm 0.4 \%$. Values ranged from $0.2 \%$ to $2.1 \%$. The lowest values were observed near the $29 \mathrm{~m}$ depth of the core.

Stable isotopic compositions of carbon of the acidified residue were similar to the samples of those from $724 \mathrm{C}$, ranging 


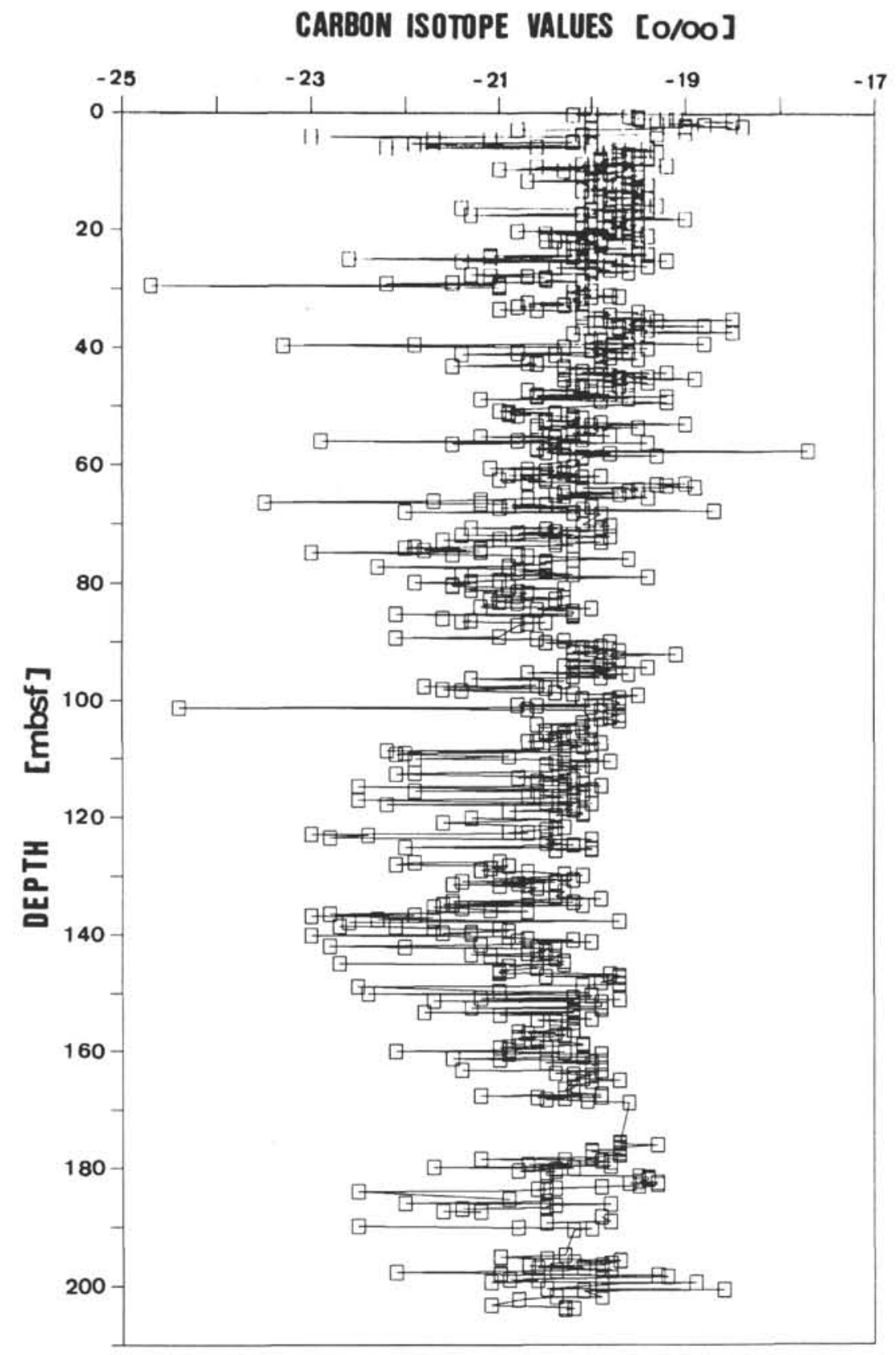

Figure 2. Site 724 core results: $\delta^{13} \mathrm{C}$ compositions.

from $-17.6 \%$ to $-25.6 \%$, with an average of $-20.1 \%$ $\pm 1.2 \%$ (Fig. 5). The excursion in percent carbon at $29 \mathrm{~m}$ to the low value of $0.2 \%$ matches closely with a change in $\delta^{13} \mathrm{C}$ to the most depleted values of the samples for this hole. There is an overall slight $(0.5 \%)$ enrichment trend toward the surface of the core.

Stable nitrogen isotopic compositions are highly variable with a general trend of decreasing enrichment toward the surface (Fig. 6). These samples averaged $11.3 \% 0 \pm 3.0 \%$, and ranged from $4.6 \%$ to $17.0 \%$. No statistically significant relationships were observed between stable nitrogen isotopic compositions and either carbon isotopes or organic carbon contents of the samples. A highly significant relationship (Pearson $\mathrm{R}=0.326$, 0.99 level) was observed between organic carbon and carbon isotopic compositions (Fig. 7).

\section{DISCUSSION}

There is no evidence of large scale input of terrigenous material into the area during Pliocene-Pleistocene times. This most likely results from the lack of major rivers that could introduce allochthonous land-derived material at great distances off the modern shoreline during periods of low sea level. The largescale effect of the monsoons on the organic production of this area appears to be small relative to inputs derived from marine production associated with upwelling. Occasional excursions of carbon and nitrogen isotopic compositions to more depleted values may result from changes in primary productivity or diagenetic processes. Further, this indicates no large-scale eolian transport of land-derived material to the area over PliocenePleistocene time. Delineation of smaller variations in isotopic 


\section{NITROGEN ISOTOPE VALUES [O\%O]}

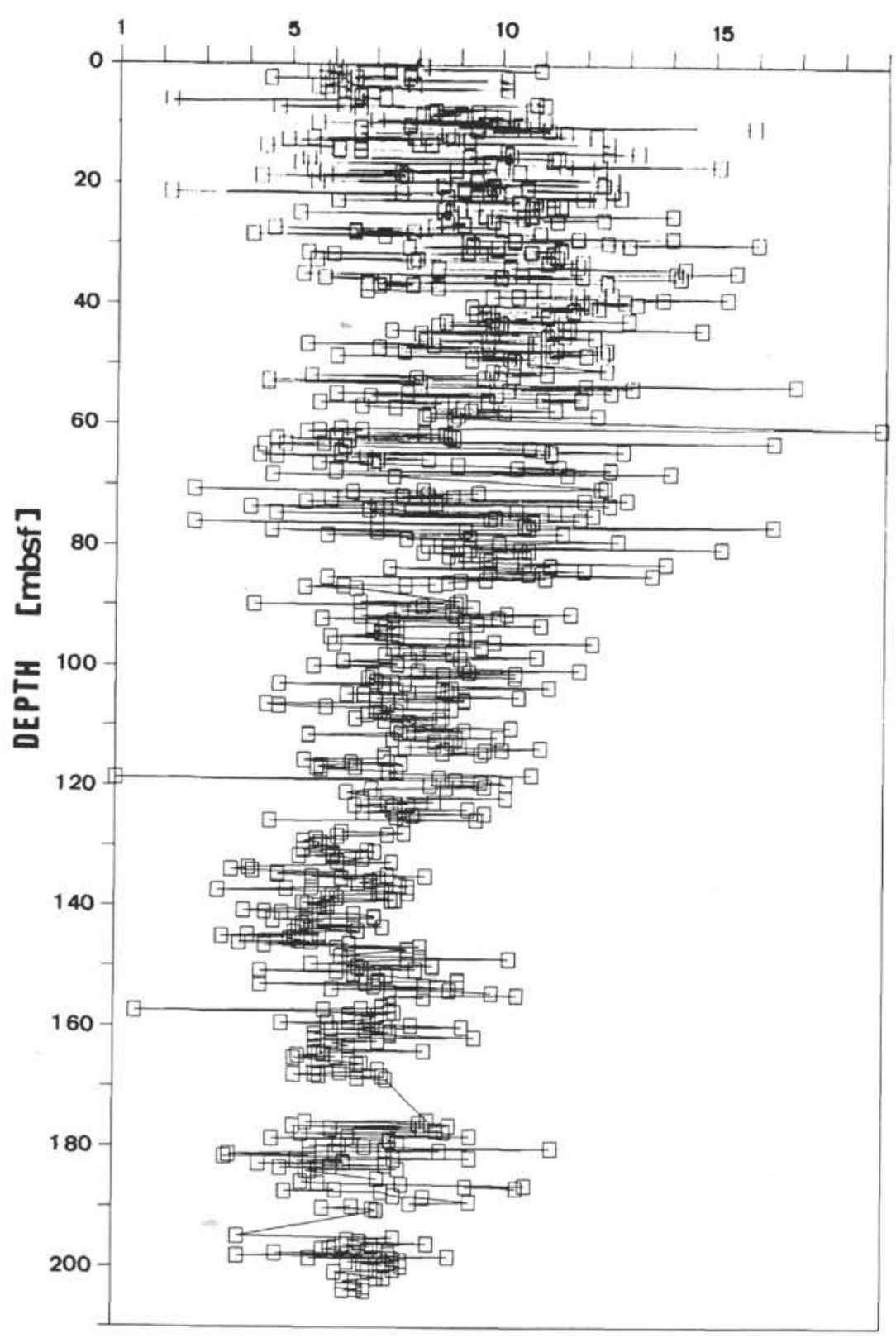

Figure 3. Site 724 core results: $\delta^{15} \mathrm{~N}$ compositions.

compositions and organic inputs awaits more detailed analysis of these samples and collaborative interpretation of the results.

During glacial periods, the areas surrounding the Arabian Sea were drier than during interglacial periods (van Campo et al., 1982; Kolla and Biscaye 1977; Sirocko, 1989 and references therein). This decrease in precipitation was coupled with the presence of shallower continental shelves and is thought to have been accompanied by diminished upwelling of deeper, cooler water. Such a setting could have caused surface water temperatures to be variable with season and dissolved carbon dioxide concentrations to be slightly enriched in ${ }^{13} \mathrm{C}$ from bicarbonate. This scenario would result in organic matter enrichments in ${ }^{13} \mathrm{C}$ during glacial periods. An increase in carbon isotopic values is noted during oxygen isotope Stage II and Stage IV as defined by Zahn and Pedersen (this volume).
Very large enrichments in stable nitrogen $(+18.9 \%)$ are found in these sediments. Nitrogen enrichments of similar magnitude have been previously reported for organic matter undergoing diagenetic reactions (Saino and Hattori, 1980). That previous study focused on a modern, deep-water ocean with a low flux of organic material to the sediments. In a situation more analogous to the present setting, the Peru Upwelling region, differences of nearly $7 \%$ have been reported in ${ }^{15} \mathrm{~N}$ for surface sediment samples only $5 \mathrm{~km}$ apart (Reimers, 1982). In areas associated with an enhanced oxygen minimum, and extensive denitrification, residual nitrate in the water column has been reported to be enriched up to $16 \%$ (Cline and Kaplan, 1975). This enrichment in ${ }^{15} \mathrm{~N}$, together with the high levels of preservation of organic matter in the sediments, could explain the heightened levels of ${ }^{15} \mathrm{~N}$ in the sediments in the present study. 


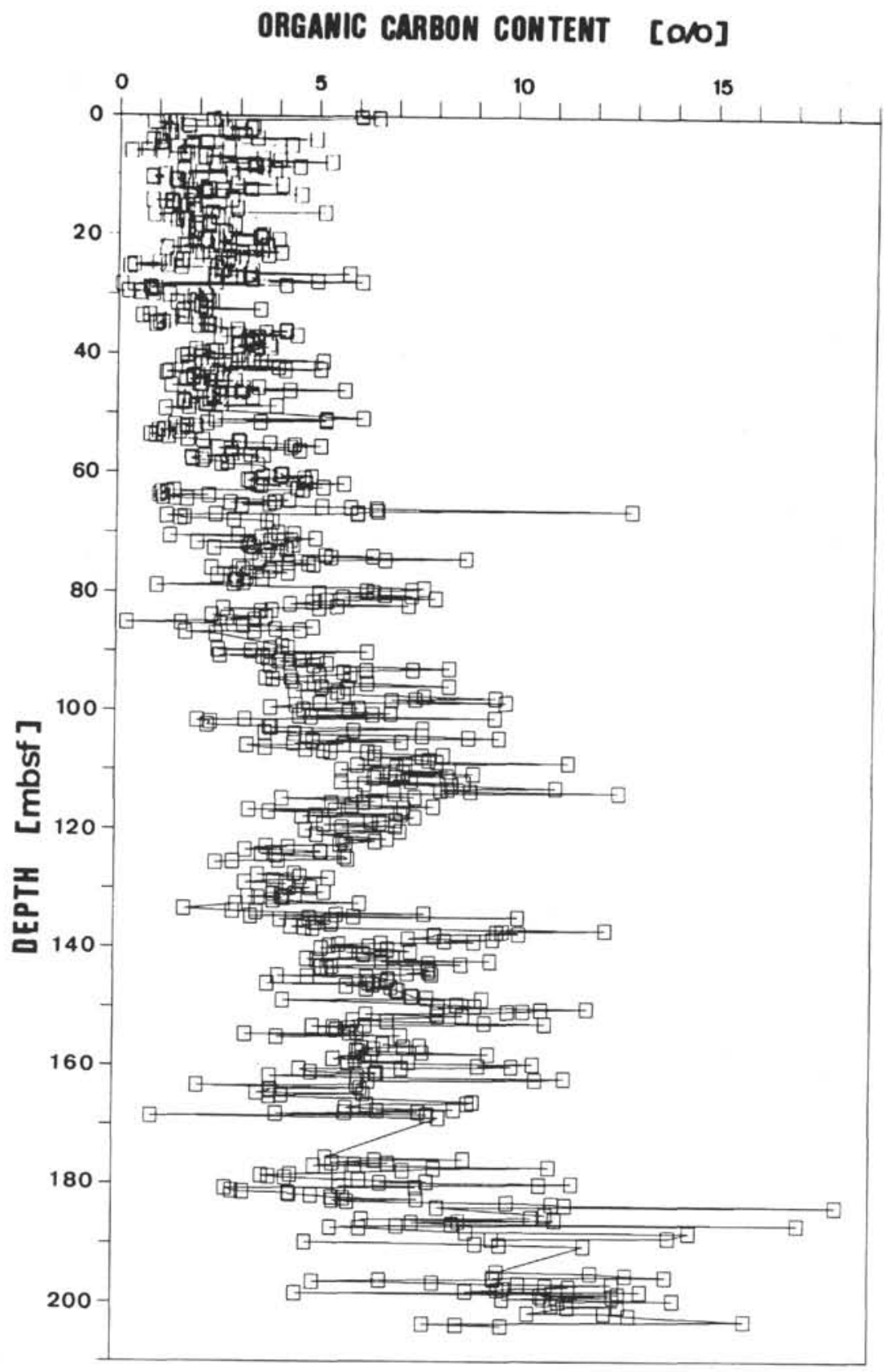

Figure 4. Site 724 core results: organic carbon content.

The $\delta^{15} \mathrm{~N}$ of sediments at Site 725 is on average about $3 \%$ more enriched than those at Site 724, suggesting that the nearer shore environment at Site $\mathbf{7 2 5}$ is more impacted by denitrification, and possibly less fluctuations in the oxygen minimum. The overall increased amount of production, or preservation of organic material, is evident at the more offshore location in the higher carbon content of the offshore sediments. It should be noted however that Shimmield et al. (in press) and Calvert and Pedersen (in press) have argued that sedimentary organic matter on the Oman Margin shows general indications of being relatively poorly preserved at the present time. The magnitude of the preserved organic material is similar to that noted by Reimers (1982) for the Peru Upwelling Area. It is of interest that the trend of enrichment in nitrogen isotopes coincides closely with a decrease in the organic carbon content of the sediments, observed following the start of the Pleistocene. This may indicate that in the Pliocene conditions in the water column were quite different than in the Pleistocene or Holocene. The production of organic matter may not have been associated with such an intense oxygen minimum as at present or in the Pleistocene, and that denitrification was not as well established.

\section{SUMMARY}

These preliminary analyses on the stable isotopic compositions of carbon and nitrogen of organic material from the western Arabian Sea indicate that:

1. The absence of depleted nitrogen and carbon isotope compositions suggests that there is little evidence for monsoon related deposits in the sediments from the Pliocene and Pleistocene at Sites 724 and 725 .

2. The preservation and/or production of organic materials in this environment appears to have changed from the Pliocene to the Pleistocene. 


\section{CARBON ISOTOPE VALUES [O/OO]}

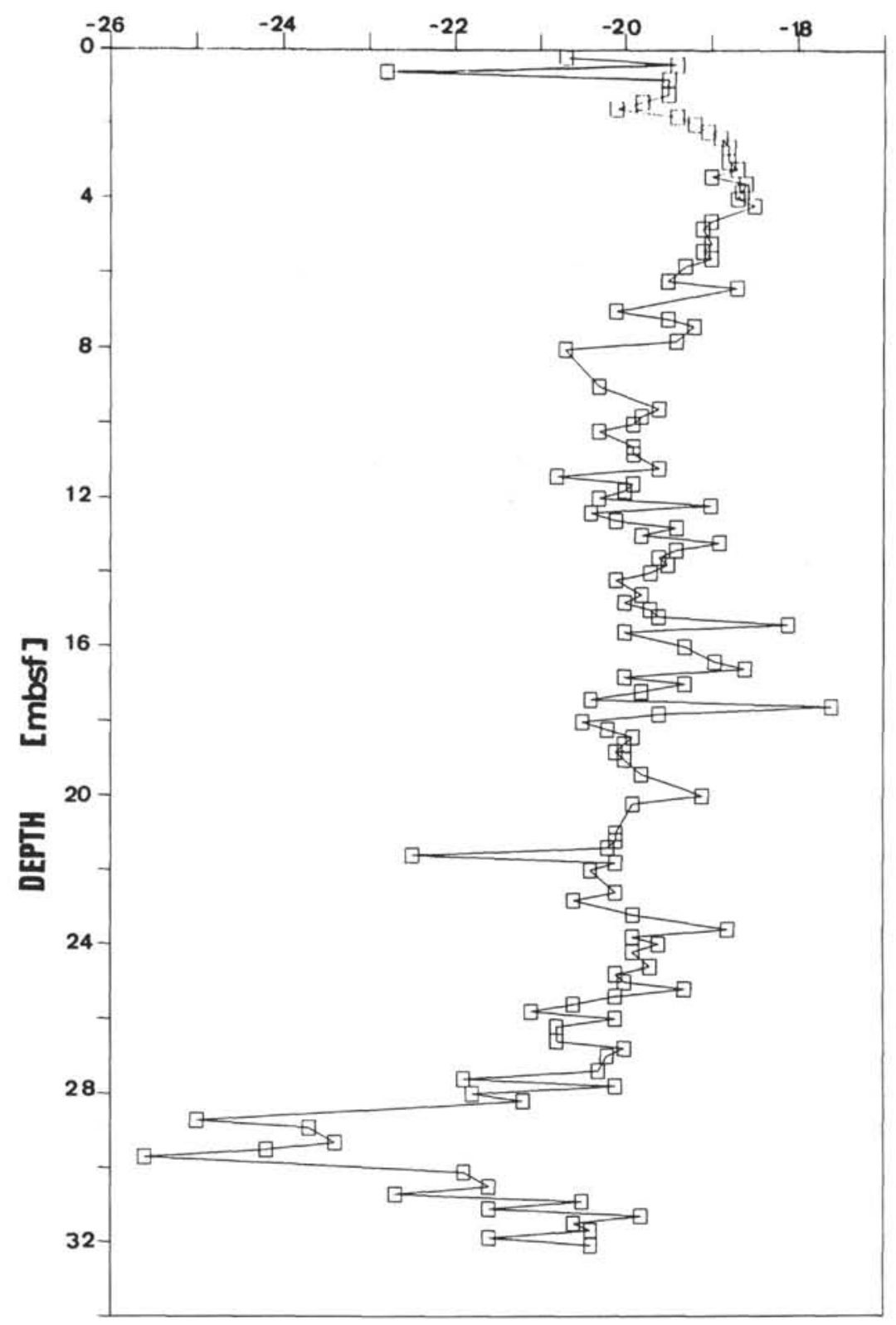

Figure 5. Site 725 core results: $\delta^{13} \mathrm{C}$ compositions.

3. The intensity of the oxygen minimum and related denitrification activity level may have also increased during the transition from the Pliocene to the Pleistocene.

4. There is a nearshore (Site 725) diminution of productivity and/or preservation, suggested by ${ }^{15} \mathrm{~N}$ compositions as compared to the offshore location (Site 724).

5. The effect of glaciation on organic matter appears to cause the carbon isotopic compositions to be slightly more enriched, especially during ice Stages II and IV.

\section{ACKNOWLEDGMENTS}

Appreciation is expressed to the crew and scientific party of ODP Leg 117 for the collection of these samples. This research was made possible by research grants from NSERC (individual operating grants and a collaborative special project) and the $\mathrm{Pe}-$ troleum Research Fund, administered by the American Chemi- cal Society (14805-AC2). The comments by two anonymous reviewers are acknowledged. The laboratory assistance of K. Pulchan, G. Hartley, P. Harrigan, T. Bieger, and N. Ostrom is greatly appreciated. We are grateful for support from the International Centre for Ocean Development for A. Muzuka and to S. Libes for providing us with documents on the Peru Upwelling Area.

\section{REFERENCES}

Altabet, M. A., and McCarthy, J. J., 1986. Vertical patterns in ${ }^{15} \mathrm{~N}$ natural abundance in PON from the surface waters of warm-core rings. J. Mar. Res., 44:185-201.

Calvert, S. E., and Pedersen, T. F., in press. Organic carbon accumulation and preservation in marine sediments: How important is anoxia? In Whelan, J. K., and Farrington, J. W. (Eds.). Productivity, Accumulation and Preservation of Organic Matter: Recent and Ancient Sediments. New York (Columbia Univ. Press). 
Cline, J. D., and Kaplan, I. R., 1975. Isotope fractionation of dissolved nitrate during denitrification in the eastern tropical North Pacific Ocean. Mar. Chem., 3:271-299.

Degens, E. T., 1969. Biogeochemistry of stable carbon isotopes. In Eglinton, G., and Murphy, M.T.J. (Eds.), Organic Geochemistry. New York (Springer-Verlag), 304-329.

Degens, E. T., Gaillard, R.R.L., Sackett, W. M., and Hellebust, J. A., 1968. Metabolic fractionation of carbon isotopes in marine plankton-I. Temperature and respiration experiments. Deep-Sea Res., 15:1-9.

Eckelmann, W. R., Broecker, W. S., Whitlock, D. W., and Allsup, J. R., 1962. Implications of carbon isotopic composition-of total organic carbon of some recent sediments and ancient oils. $A A P G$ Bull., 46:699-704.

Emerson S., and Hedges, J. I., 1988. Processes controlling the organic carbon content of open ocean sediments. Paleoceanography, 3:621634.

Fontugne, M. R., and Duplessy, J. C., 1978. Carbon isotope ratio of marine plankton related to surface water masses. Earth Planet. Sci. Lett., 41:365-371.

, 1981. Organic carbon isotopic fractionation by marine plankton in the temperature range -1 to $31^{\circ} \mathrm{C}$. Oceanol. Acta, 4:85-90.

Gearing, P., Plucker, P. F., and Parker, P. L., 1977. Organic carbon stable isotope ratios of continental margin sediments. Mar. Chem., 5: 251-256.

Hedges, J. I., and Parker, P. L., 1976. Land derived organic matter in surface sediments from the Gulf of Mexico. Geochim. Cosmochim. Acta, 40:1019-1029.

Kolla, V., and Biscaye, P., 1977. Distribution and origin of quartz in the sediments of the Indian Ocean. J. Sediment. Petrol., 47:642-649.

Libes, S. M., and Deuser, W. G., 1988. The isotope geochemistry of particulate nitrogen in the Peru Upwelling Area and the Gulf of Maine. Deep-Sea Res., 35:517-533.

Liu, K. K., 1979. Geochemistry of inorganic nitrogen compounds in two marine environments: the Santa Barbara Basin and the ocean off Peru. [Ph.D. dissert.] Univ. California, Los Angeles.

Macko, S. A. 1989. Stable isotope organic geochemistry of sediments from the Labrador Sea (Sites 646 and 647) and Baffin Bay (Site 645), ODP Leg 105. In Srivastava, S. P., Arthur, M., et al., Leg 105: Proc. ODP, Sci. Results, College Station, TX, 209-231.

Macko, S. A., Entzeroth, L., and Parker, P. L., 1984. Regional differences in nitrogen and carbon isotopes on the continental shelf of the Gulf of Mexico. Naturwissenschaften, 71:374-375.

Macko, S. A., Estep, M.L.F., Hare, P. E., and Hoering, T. C., 1987a. Isotopic fractionation of nitrogen and carbon in the synthesis of amino acids by microorganisms. Chem. Geol. 65:79-92.

Macko, S. A., and Pereira, C.P.G., 1990. Neogene paleoclimate development of the Antarctic Weddell Sea region: organic geochemistry. In Barker, P. F., Kennett, J. P., et al., Leg 113: Proc. ODP, Sci. Results, College Station, TX, 881-897.

Macko, S. A., Pulchan, K., and Ivany, D. E., 1987b. Organic geochemistry of Baffin Island fjords. Sedimentology of Arctic fjords experiment. Geol. Surv. Can. Open-File Rept., 1589:1-34.

Muller, P. J., Erlenkeuser, H., and von Grafenstein, R., 1983. Glacialinterglacial cycles in oceanic productivity inferred from organic carbon contents in eastern North Atlantic sediment cores. In Suess, E., and Thiede, J. (Eds.) Coastal Upwelling, Part B. Sedimentary Records of Ancient Coastal Upwelling. New York (Plenum Press), 365398.

Muller, P. J., and Suess, E., 1979. Productivity, sedimentation rate, and sedimentary carbon content in the oceans. 1. Organic carbon preservation. Deep-Sea Res., 26A:1347-1362.

Newman, J. W., Parker, P. L., and Behrens, E. W., 1973. Organic carbon isotope ratios in Quaternary cores from the Gulf of Mexico. Geochim. Cosmochim. Acta, 37:225-238.
Parker, P. L., Behrens, E. W., Calder, J. A., and Shultz, W., 1972. Stable carbon isotope ratio variations in the organic carbon from Gulf of Mexico sediments. Contrib. Mar. Sci., 16:139-147.

Peters, K. E., Sweeney, R. E., and Kaplan, I. R. 1978. Correlation of carbon and nitrogen stable isotope ratios in sedimentary organic matter. Limnol. Oceanogr., 23:598-604.

Prell, W. L., and Curry, W. B., 1981. Faunal and isotopic indices of monsoonal upwelling: Western Arabian Sea. Oceanol. Acta, 4:9198.

Prell, W. L., and Kutzbach, J. E., 1987. Monsoon variability over the past 150,000 years. J. Geophys. Res., 92:8411-8425.

Prell, W. L., and van Campo, E., 1986. Coherent response of Arabian Sea upwelling and pollen transport to the late Quaternary monsoonal winds. Nature, 323:526-528.

Reimers, C., 1982. Sedimentary organic matter: distribution and alteration processes in the coastal upwelling region off Peru. [Ph.D. dissert.] Oregon State Univ.

Reimers, C., and Suess, E., 1983. Spatial and temporal patterns of organic matter accumulation on the Peru continental margin. In Suess, E., and Thiede, J. (Eds.) Coastal Upwelling, Part B. Sedimentary Records of Ancient Coastal Upwelling. New York (Plenum Press), 311-345.

Romankevich, E. A., 1984. Geochemistry of Organic Matter in the Ocean. New York (Springer-Verlag).

Rossignol-Strick, M., 1983. African monsoons, an immediate climate response to orbital insolation. Nature, 304:46-49.

Sackett, W. M., Eckelmann, W. R., Bender, M. L., and Be, A.W.H., 1965. Temperature dependence of carbon isotope compositions in marine plankton and sediments. Science, 148:235-237.

Sackett, W. M., and Thompson, R. R., 1963. Isotope organic carbon composition of Recent continental derived clastic sediments of Eastern Gulf Coast, Gulf of Mexico. AAPG Bull., 147:535-531.

Saino, T., and Hattori, A., 1980. ${ }^{15} \mathrm{~N}$ natural abundance in oceanic suspended particulate matter. Nature, 283:752-754.

Shimmield, G. B., Price, N. B., and Pedersen, T. F., in press. The influence of hydrography, bathymetry and productivity on sediment type and composition on the Oman Margin and in the Northwest Arabian Sea. Geol. Soc. Spec. Pap.

Sigleo, A. C., and Macko, S. A., 1985. Stable isotope and amino acid composition of estuarine dissolved colloidal material. In Sigleo, A. C., and Hattori, A. (Eds.). Marine and Estuarine Geochemistry. Chelsea, MI (Lewis Publishers Inc.) 30-48.

Sirocko, F., 1989. Accumulation of eolian sediments in the northwest Indian Ocean: record of the climatic history of Arabia and India. Repts. Geol. Inst. Kiel, 27:1-185 (in German).

ten Haven, L., Farrimond, P., Poynter, J. G., Rullkotter, J., Eglinton, G., and Welte, D. H., 1989. Variations in the composition of extractable lipids in coastal sediments underlying active upwelling regimes. A study from ODP Legs 108, 112, and 117. 14th Internat. Mtg. on Org. Geochem. Paris (Abstract).

van Campo, E., Duplessy, J. C., and Rossignol-Strick, M., 1982. Climatic conditions deduced from 150-kyr oxygen isotope pollen record from the Arabian Sea. Nature, 296:56-59.

Wada, E. 1980. Nitrogen isotope fractionation and its significance in biogeochemical processes occurring in marine environments. In Goldberg, E. D., and Horibe, Y. (Eds.). Isotope Marine Chemistry. Uchida Rokakuho Pub., Tokyo, 375-398.

Zieman, J. C., Macko, S. A,. and Mills, A. L., 1984. Role of seagrasses and mangroves in estuarine foodwebs: temporal and spatial changes in stable isotope composition and amino acid content during decomposition. Bull. Mar. Sci., 35:380-392.

Date of initial receipt: 9 October 1989

Date of acceptance: 20 February 1990

Ms 117B-163 


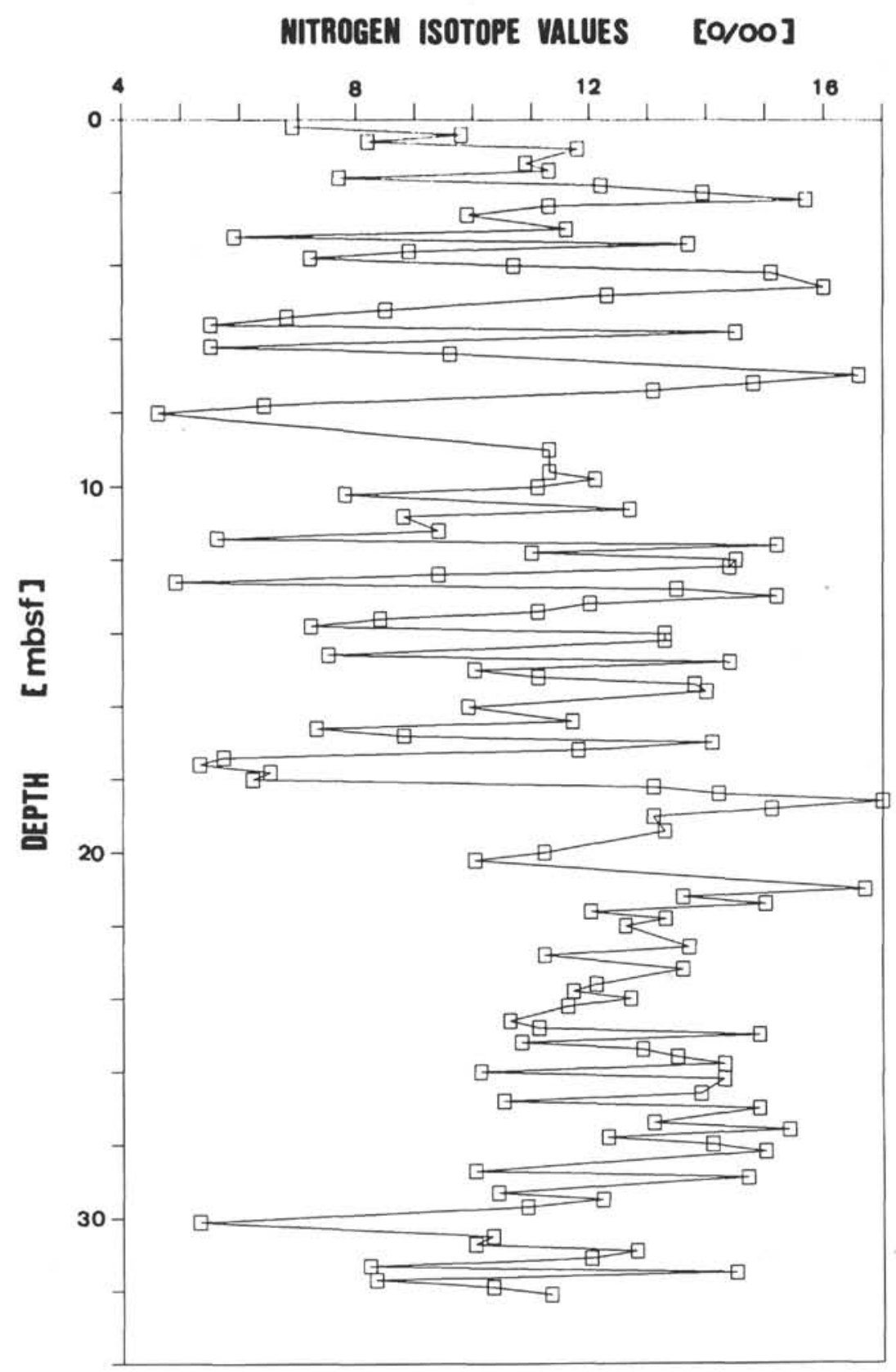

Figure 6. Site 725 core results: $\delta^{15} \mathrm{~N}$ compositions. 
A.N.N. MUZUKA, S. A. MACKO, T. F. PEDERSEN

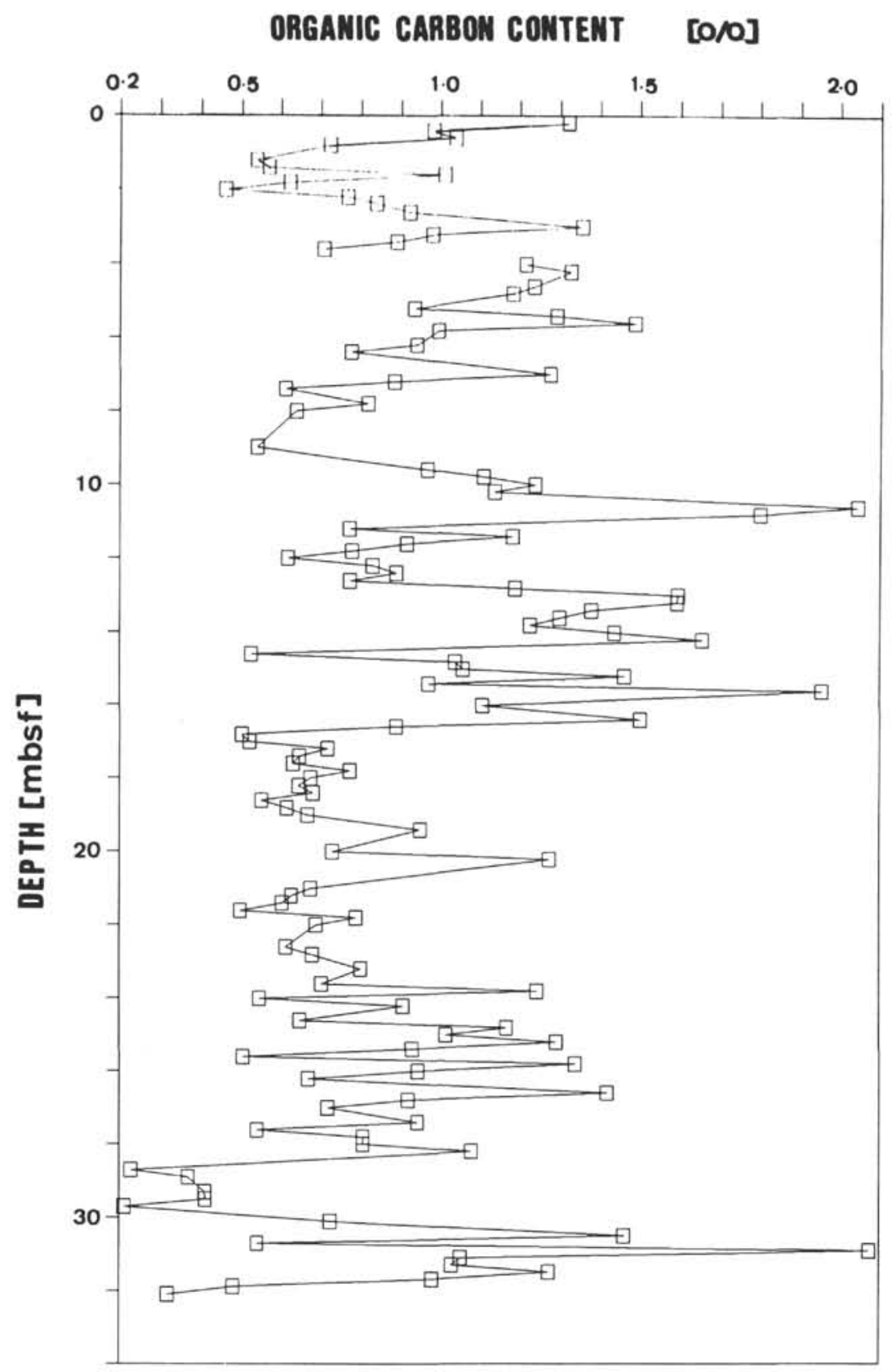

Figure 7. Site 725 core results: organic carbon content. 